\title{
Le sort des migrants africains en Chine : \\ L' afrophobie entrave la course de la Chine pour les ressources et les marchés de l'Afrique
}

\author{
Dirk Kohnert ${ }^{1}$
}

Cas d'un dessin animé chinois afrophobe

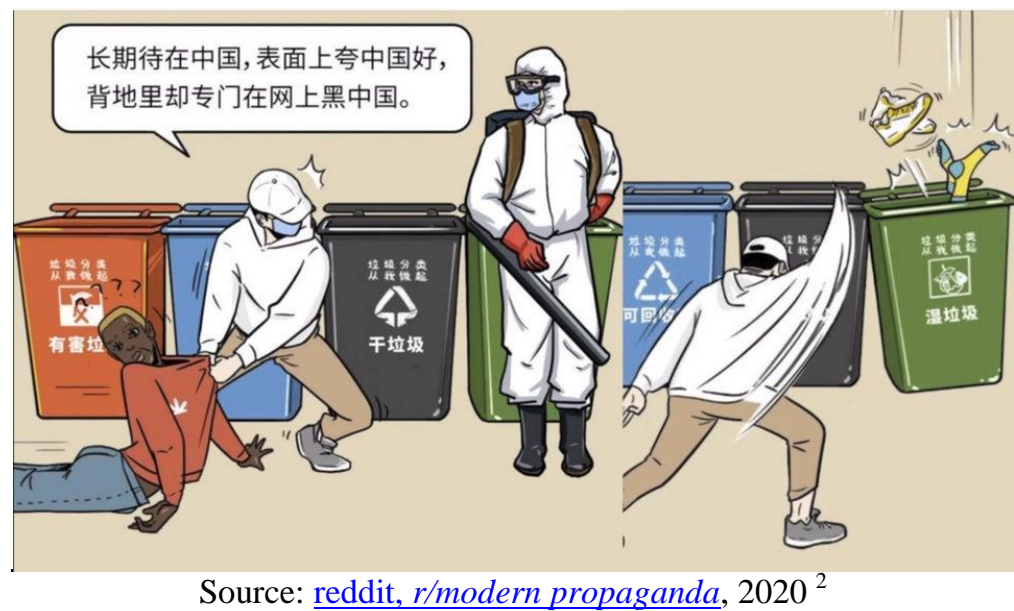

Résumé: La discrimination à l'encontre des quelque 500000 immigrés africains (pour la plupart irréguliers) s'est récemment répandue en Chine. Pendant la pandémie de corona, cela dégénère en une véritable afrophobie. Peu de temps auparavant, cinq Nigérians de Guangzhou auraient été testés positifs pour Covid-19. Les Africains sont largement accusés d'être des trafiquants de drogue et des criminels. En outre, ils mettraient en danger la compétitivité mondiale de la Chine pour les ressources de l'Afrique par le biais du dénigrement médiatiques à l'étranger. Les rapports actuels témoignent du déplacement des migrants africains des maisons et des hôtels de Guangzhou (Canton), où vivent la plupart des Africains. Ils dépendent de réseaux informels, pour la plupart illégaux, pour pouvoir rester dans le pays. Dans les réseaux sociaux en ligne, l'afrophobie et le cyber-racisme sont particulièrement prononcés. Ainsi, le racisme est plus profondément enraciné dans la mentalité de nombreux Chinois qu'on ne le pense généralement. Selon un proverbe chinois traditionnel, le plus grand mal à éviter est « la nation détruite et la race anéantie ». En outre, depuis 2005, l'accaparement des terres par des entrepreneurs chinois en Afrique subsaharienne a attiré l'attention de la communauté internationale. Son objectif principal est d'assurer la sécurité alimentaire en Chine et de profiter de la spéculation céréalière internationale. Il a été légitimé racialement dès le départ, avec des slogans tels que, seuls les investissements chinois pouvaient sauver les Africains de leur «paresse » traditionnelle.

Mots-clés: Chine, Afrique, relations sino-africaines, migration internationale, xénophobie, afrophobie, racisme, violence politique, BRICS, secteur informel, immigration illégale, migration forcée, minorités, envois de fonds

JEL-code: F16, F22, F24, F51, F54, I24, I31, J46, J61, N15, N35, O15, O17, O53, Z13

\footnotetext{
${ }^{1}$ Dirk Kohnert, directeur adjoint (retraité), Institut des affaires africaines, GIGA, Hambourg. Projet : 6 janvier 2022.

2 Posté par u/friend1y, 2020, dénonçant un dessin animé chinois anti-étrangers afrophobe et raciste intitulé : " Rester longtemps en Chine; faire l'éloge de la Chine devant les gens tout en parlant de la Chine sur Internet dans le dos de tout le monde "... OEPEQY renvoie à un "contexte supplémentaire: le mot pour «salir (quelqu'un)», traduit ici par « dire de la merde », est 黑, qui signifie aussi «noir». Cela peut être une forme de jeu de mots racial (Source: OEPEQY, reddit, r/modern propaganda, 2020). -- Le dessin fait référence à la politique barbare des propriétaires fonciers et des municipalités chinoises pour expulser les locataires africains de leurs appartements après avoir enduré des mois de verrouillage (Asiedu, 2020).
} 


\section{Introduction}

La dure stratégie chinoise "Zéro COVID » a été saluée internationalement comme un modèle de gestion de la pandémie de COVID-19 malgré la fermeture des frontières, les blocages récurrents et les interruptions des activités économiques et sociales (AlTakarli, 2020). Néanmoins, les citoyens chinois ordinaires ne s'inquiétaient apparemment pas du maintien de mesures anti-virus strictes tant qu'ils se sentaient en sécurité (McDonell, 2021). Cependant, les quelque 500000 Africains en Chine qui auraient été ciblés par les autorités locales et les résidents pendant la pandémie ont fortement souffert des attaques xénophobes de plus en plus racistes de la population, comme le montrera ce qui suit. La plupart d'entre eux vivaient et travaillaient à Guangzhou, la métropole de la rivière des Perles dans le sud de la Chine, anciennement appelée Canton, à environ $130 \mathrm{~km}$ au nord-nord-ouest de Hong Kong. C'est pourquoi la ville a également été surnommée «Petite Afrique » ou « La ville du chocolat » où environ 150000 Africains vivaient, des résidents de longue durée, selon les statistiques gouvernementales de 2015, mais plus probablement jusqu'à 300 000, y compris les nombreux immigrants irréguliers (illégaux) (Kuo, 2016).

Peu de temps après les attaques xénophobes contre les migrants africains à Guangzhou, les autorités locales ont publié une lettre ouverte menaçant la tolérance zéro sur le racisme et le sexisme après des cas de discrimination de ressortissants africains (pandémie de COVID-19 en Chine, Wikipédia). Ici, et dans ce qui suit, le concept théorique de «race» et de " racisme " est compris, à la suite de Robert Castillo, comme inextricablement lié à l'histoire euro-américaine et la construction de la « race » en tant qu'élément intégral de la « race » en tant qu'élément intégral de la compréhension globale coloniale et postcoloniale des groupes ethniques, des identités et du nationalisme (Castillo, 2020; race humaine, Wikipédia).

La plupart des Africains sont venus dans le pays au cours du commerce florissant de la Chine en Afrique subsaharienne (ASS) au début des années 2000 et de l'afflux concomitant de petits commerçants africains en Chine (Marfaing \& Thiel, 2015 ; Zhou, et al., 2016). ; 2016a ; Giese \& Marfaing, 2019). Faute de données fiables sur le nombre réel de migrants africains en Chine, la présence chinoise croissante en Afrique pourrait être considérée comme un indicateur approximatif ou une variable «dummy» de l'augmentation de la migration africaine vers la Chine également, à côté de l'évolution de l'investissement et commerce africains de la Chine. Ce dernier a continué de prospérer jusqu'à la pandémie, atteignant US\$ 192 milliards en 2019, avec environ un à deux millions de Chinois vivant en Afrique (Cissé, 2021).

Néanmoins, il existait de longue date des sentiments anti-africains au sein de la population chinoise, voire parmi les migrants chinois en Afrique, équivalant au racisme et à une véritable afrophobie, i.e. un racisme anti-noir, pendant la pandémie. Dans les médias sociaux, des clips vidéo sont devenus viraux montrant des Africains, principalement du Nigeria, du Ghana, du Kenya et de l'Ouganda, battus, expulsés de leurs appartements, refusés l'entrée dans les hôtels, et même dans les magasins de plats à emporter McDonald's. Les gouvernements africains, l'Union africaine (UA) et les États-Unis ont protesté avec véhémence et tenté de faire pression sur Pékin (Beijing, 北京) pour qu'il mette fin à ces attentats, mais essentiellement en vain (Asiedu, 2020). Selon Liu Junhai de l'Université Renmin de Chine, il y a toujours un manque de sensibilisation du public aux questions raciales en Chine. Un cas particulièrement flagrant s'est produit en 2016, lorsqu'une publicité pour un détergent à lessive chinois a déclenché un tollé en montrant une femme mettant un homme noir dans une machine à laver qui est ressorti comme un Chinois à la peau claire après un lavage (PTI, 2016). 
Graphique 1 : Afflux de migrants chinois en Afrique, $2000-2019^{3}$

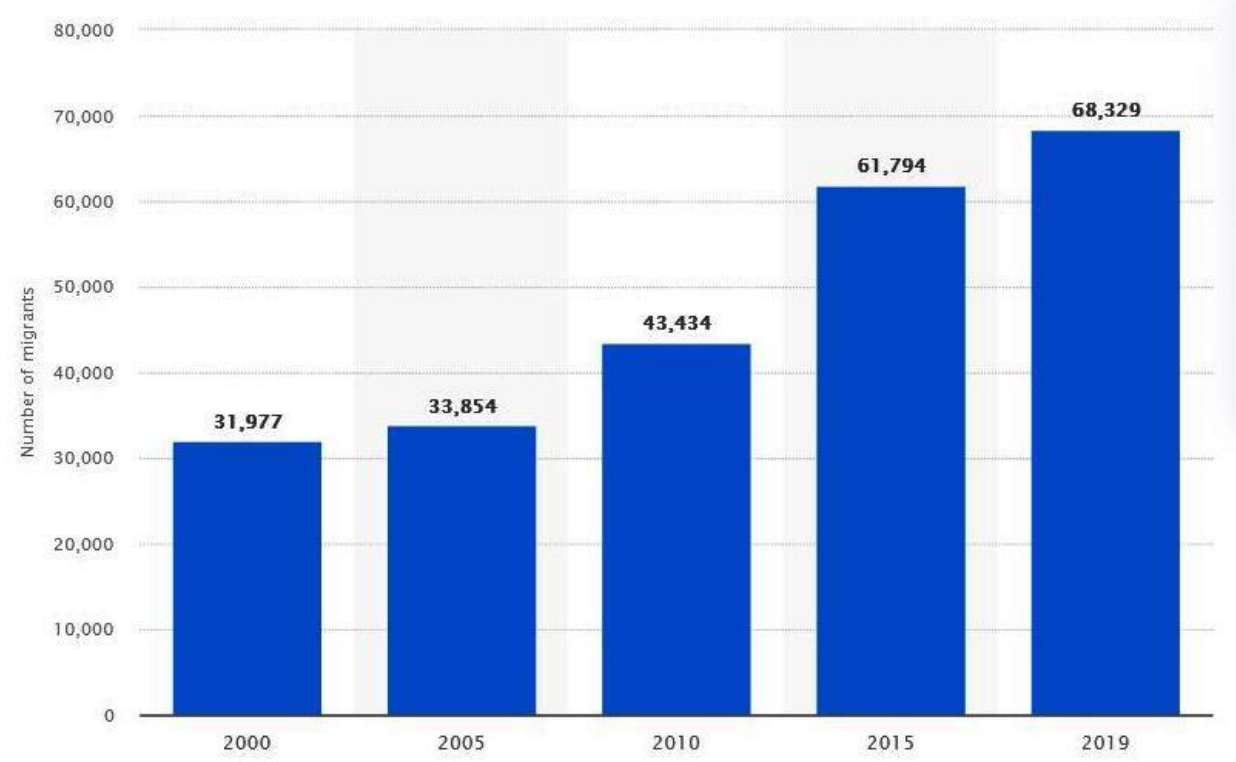

Source: Sasu, 2021; (C) Statista 2021

Graphique 2 : les Chinois comptent parmi les 3 premiers pays d'origine des migrants à l'étranger (en millions, 2019)
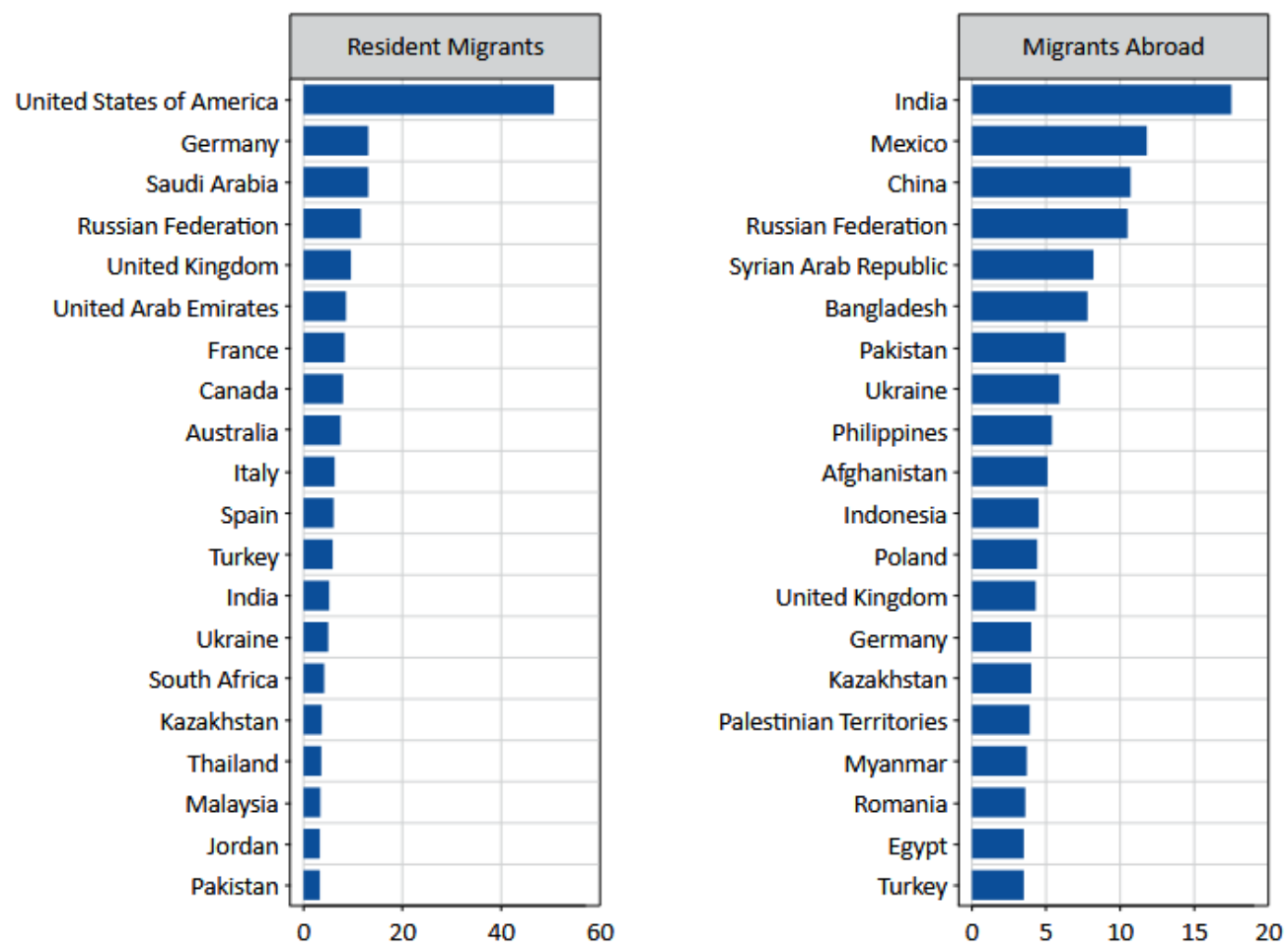

Source: UN DESA, 2019a (accessed 18 September 2019).

Source: UN-IOM, World migration report, 2020, p. 26.

\footnotetext{
${ }^{3}$ Ces chiffres officiels sont probablement largement sous-estimés en raison du titre irrégulier (illégal) (absence de titre de séjour) de nombreux migrants chinois en Afrique au statut précaire. Par conséquent, ces chiffres pourraient refléter plutôt le sens de la croissance.
} 
Dans ce qui suit, une brève description de l'histoire des relations sino-africaines conduira à l'analyse du tissu sous-jacent de l'afrophobie croissante en Chine et de ses conséquences sur la position de Pékin dans la compétition mondiale pour les ressources et les marchés africains. Ainsi, nous devons toujours garder en mémoire que l'opposition locale à la migration et aux migrants est courante globalement, y compris dans de nombreux pays de l'UE et aux ÉtatsUnis.

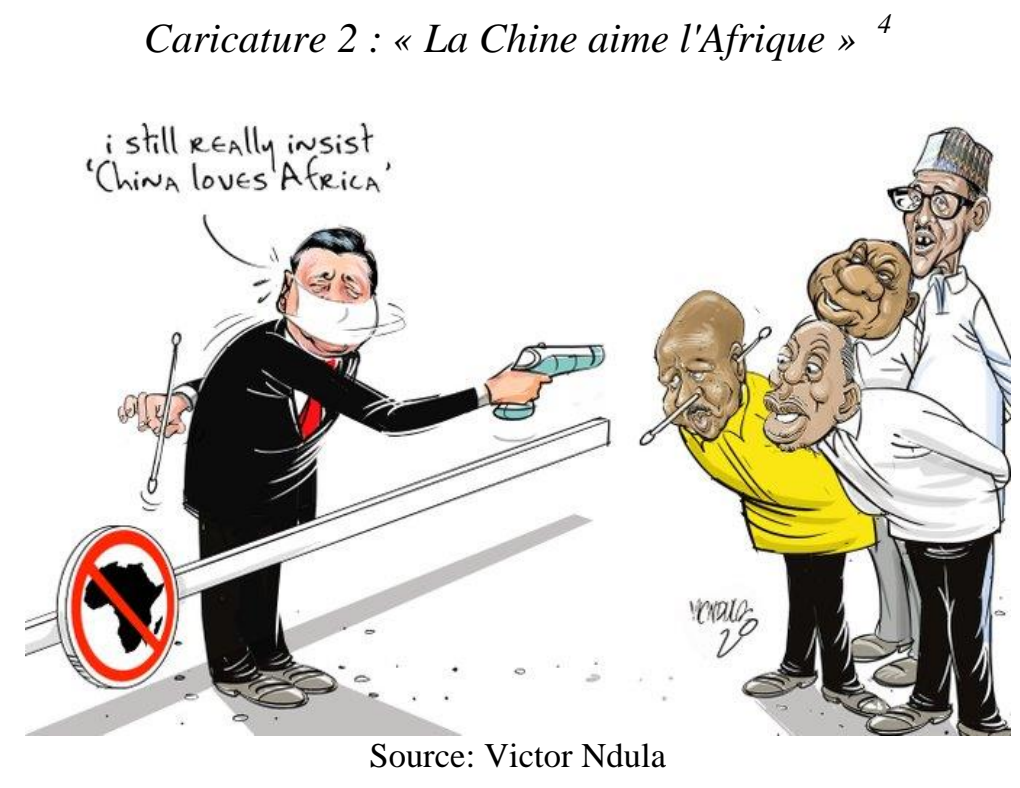

Les questions centrales d'analyse seront la question de savoir si la société chinoise, en général, a des tendances racistes vis-à-vis des étrangers et/ou des minorités, et si les actions et réactions de l'administration du pays à tous les niveaux, mais notamment dans les centres urbains, avait fait partie du problème.

\section{Histoire succincte des relations et du commerce sino-africains}

Les premiers enregistrements des relations et du commerce sino-africains remontent aux premiers jours de la route de la soie chinoise au ler siècle après $\mathrm{AD}$, aux voyages des explorateurs marocains et somaliens au 14ème siècle et aux rencontres ultérieures d'Africains et de Chinois au cours des voyages du 15ème siècle de l'amiral chinois Zheng He et de sa flotte sous le règne de la dynastie Ming. La flotte a atteint la côte africaine de la Somalie et l'a suivie jusqu'au canal du Mozambique. Les premières preuves du commerce sino-africain sont des perles et de la porcelaine chinoise découvertes à Grand Zimbabwe (Africa-China relations, Wikipédia; Lan, 2017).

La Chine moderne d'après-guerre a forgé de solides relations étrangères avec les gouvernements africains à l'époque de la guerre froide dans le cadre de la lutte anticoloniale et du développement du mouvement des non-alignés depuis la Conférence de Bandung en 1955.

\footnotetext{
4 Le président chinois Xi Jinping, menaçant des hommes d'État africains, parmi lesquels le président nigérian Muhammadu Buhari, le président ougandais Yoweri Museveni et le chef de l'État kenyan Uhuru Kenyatta Source: Cartoon posted by Victor Ndula, Twitter, @ndula_victor, 12 April 2020, Cartoon for @ dailynation, \#china \#Africa \#covid19 \#xenophobia ... 'the irony of it all'. Consulté le 20 décembre 2021.
} 
Les gouvernements participants visaient à promouvoir la coopération politique, économique et culturelle afro-asiatique (Servant, 2005; Zeleza, 2008; Africa-China relations, Wikipédia).

Carte 1 : routes commerciales autour de la « $\underline{\text { route de la soie } » ~ c h i n o i s e ~ d u ~ 1 e r ~ s i e ̀ c l e ~ A D . ~}$

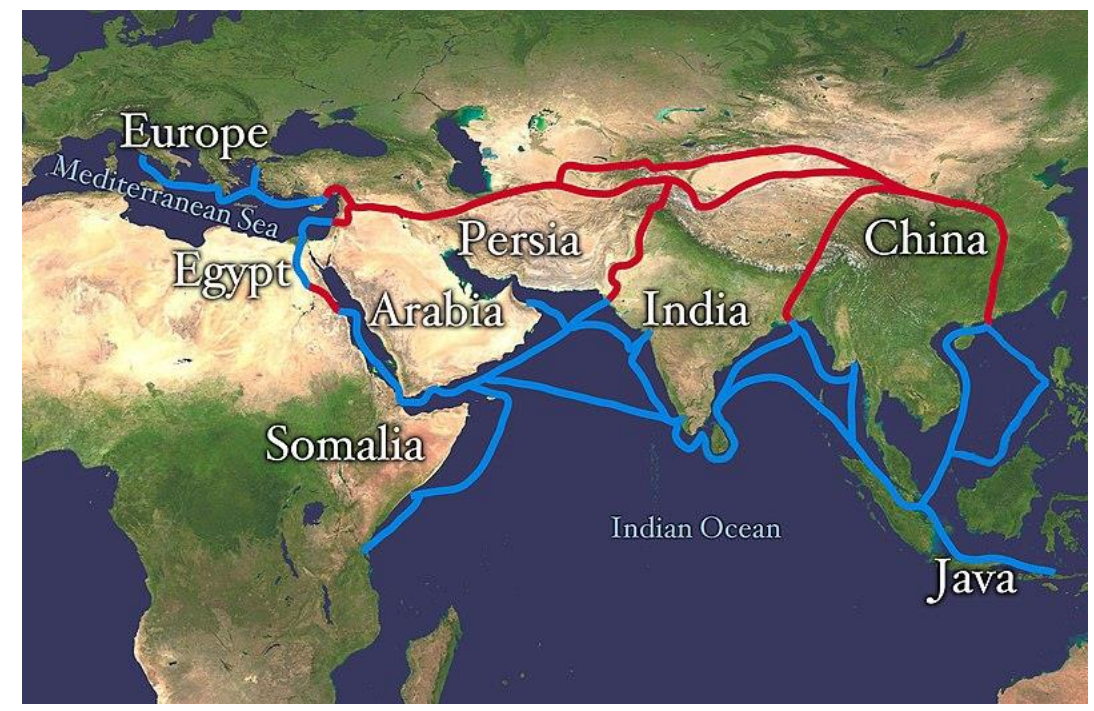

Source: Africa-China relations, Wikipedia

Le ressentiment massif contre les Africains est devenu publiquement visible dans les années 1960, lorsque de nombreux étudiants africains de pays africains amis de la Chine sont venus en Chine pour étudier à Pékin, et depuis les années 1970 également en dehors de la capitale (Pomfret, 1989; Lufrano, 1992).

Photo 1 : Manifestation chinoise contre les étudiants africains à Pékin, $1986^{5}$

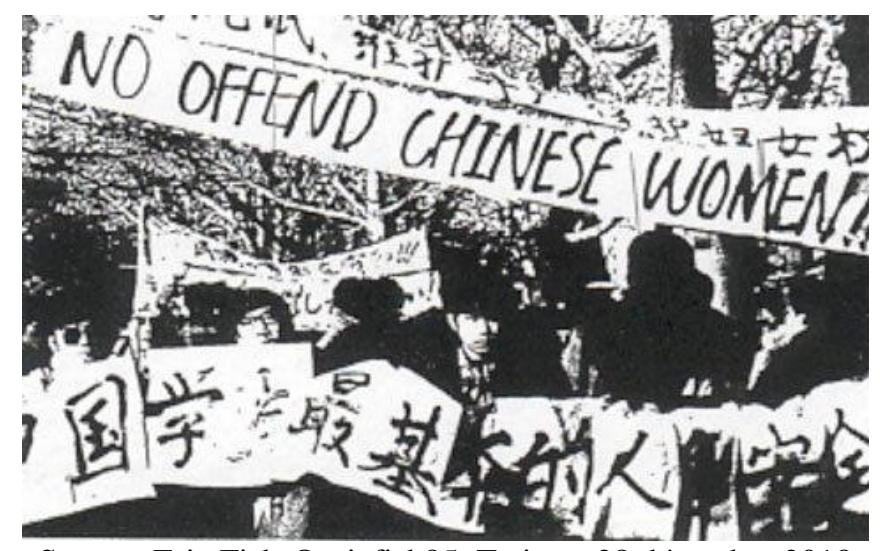

Source: Eric Fish @ericfish85, Twitter, 28 décembre 2018

Les ressentiments ont été justifiés par les étudiants chinois en raison de la position privilégiée de nombreux étudiants africains qui ont reçu des bourses d'études plus importantes que les étudiants chinois résidents. En outre, l'hostilité des étudiants chinois envers les Africains a éclaté en raison des agressions contre les Africains qui rivalisaient avec la population locale pour des relations amicales avec les femmes chinoises. Même les manifestations de la place

\footnotetext{
${ }^{5}$ Eric Fish, @ericfish85. --- «Les manifestations anti-africaines ont fait sortir des étudiants dans les rues pour protester contre les autorités d'une manière qui a ouvert la voie aux manifestations de Tian'anmen 4 mois plus tard. En fait, certains organisateurs de manifestations anti-africaines à Pékin auraient également organisé pendant le mouvement Tian'anmen ». http://cowriesrice.blogspot.com/2012/12/on
} 
Tian'anmen de 1989 contenaient des références à des sentiments anti-africains, visibles sur des banderoles proclamant "Arrêtez de profiter des femmes chinoises », même si la grande majorité des étudiants africains avaient déjà quitté le pays à ce moment-là (Nanjing AntiAfrican protests, Wikipedia).

Le racisme de l'étudiant chinois a montré des différences significatives avec le cyber-racisme chinois anti-africain qui reflète aujourd'hui la pensée chinoise (Cheng, 2011). Ce dernier est basé sur l'idéologie d'une infériorité raciale noire présumée, largement partagée par les Chinois qui considèrent qu'il est mauvais pour les Africains de créer des problèmes sociaux dans les villes chinoises et d'entraver ainsi l'agence de la Chine en Afrique (Mohan \& Lampert, 2013; Kohnert, 2016; 2010). Pourtant, la mentalité sous-jacente est plus profondément enracinée chez la plupart des Chinois qui partagent un dicton commun selon lequel le plus grand mal à éviter serait la «nation détruite et la race anéantie » (wangguo miezhong, 亡国灭种) (Cheng, 2011). L' «accaparement des terres » des migrants et des entreprises chinois en Afrique depuis 2005, et la fondation de colonies agricoles connues sous le nom de villages Baoding, créées dans plus de deux douzaines de pays africains étaient intrinsèquement racistes et promouvaient l'idéologie selon laquelle les investissements chinois pourraient « sauver» les Africains de leur «paresse» (Liu, 2018; Perrot \& Malaquais 2009).

Caricature 3 : «Bienvenue à Chafrica » Regard africain sur l'omniprésence des Chinois en Afrique ${ }^{6}$

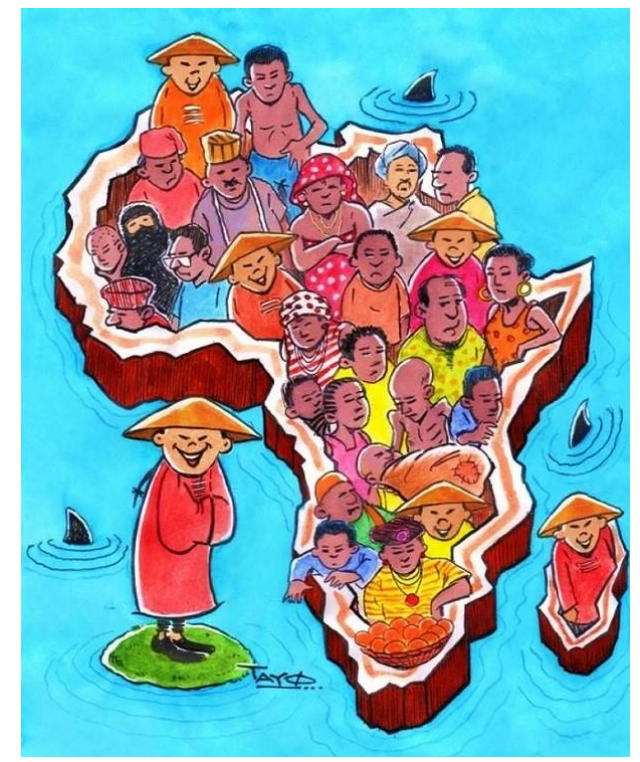

Source: Camille Bour, 24 décembre 2021

De ce fait, il a répété des préjugés coloniaux européens profondément enracinés et créé une sorte de nouveau colonialisme de colonsde dans une "Afrique sauvage et non civilisée » associée à un fort sentiment de supériorité raciale (Cheng, 2011). Cela contrastait sensiblement avec l'histoire chinoise de l'après-guerre, lorsque Mao Tse Tung aurait remercié les «frères noirs » africains d'avoir voté pour l'accès de la Chine en 1971 aux Nations Unies

\footnotetext{
${ }^{6}$ Dans ce dessin, le célèbre dessinateur nigérian Tayo Fatunla «a voulu illustrer l'omniprésence de Pékin sur le continent». «Bienvenue à Chafrica » - Pour certains observateurs, l'influence de la Chine est un réel avantage pour l'Afrique. Selon le caricaturiste nigérian Tayo Fatunla, «c'est une relation saine qui peut fonctionner parce que les Nigérians ont accepté l'aide de la Chine, là où d'autres ont échoué ». «Il y a aussi beaucoup d'Africains en Chine, donc la compréhension est mutuelle ». «Bienvenue à CHAFRICA », explique Tayo Fatunla. (Camille Bour, Paris: tv5monde.com 24, 20 décembre 2021).
} 
(Cheng, 2019). Cependant, cette version de l'histoire a été réfutée avec véhémence par les universitaires chinois comme une fausse histoire en 1999. Parmi les quarante-deux États africains membres de l'ONU, vingt-six avaient voté en faveur de la reconnaissance de la République populaire de Chine en tant que seul représentant de la Chine dans le Résolution 2758 de l'Assemblée générale des Nations Unies d'octobre 1971. Dont six pays arabes d'Afrique du Nord et donc non «noirs ». Seize nations africaines (la majorité d'entre elles noires) avaient même voté contre la RP Chine ${ }^{7}$ (Cheng, 2019). Ainsi, l'afrophobie chinoise, et le racisme en général, reflétaient dans les faits les problèmes et les aspirations de la Chine par la représentation de l'Afrique et des Noirs comme étrangers. Cette arrogance était déjà héritée de la vision traditionnelle du monde sinocentrique qui associait les étrangers à la peau foncée à un statut social non libre à la hiérarchie raciale d'aujourd'hui (Cheng, 2019).

La ruée des petits commerçants chinois vers l'Afrique a suivi la grande poussée du commerce et des investissements chinois en Afrique depuis environ 2005 (Rotberg, 2017). Cependant, les fondations avaient été posées bien avant à la fin des années 1980, lorsque l'augmentation des flux des migrantes entre la Chine et l'Afrique avait favorisé les relations bilatérales, largement basées sur l'agence africaine (Mohan \& Lampert, 2013 ; Kohnert, 2016 ; 2010). Cette tendance s'était manifestée par la création du Forum sur la coopération sino-africaine (FOCAC) en 2000. L'essor des relations bilatérales de commerce et d'investissement a créé des opportunités prometteuses pour l'emploi et d'autres possibilités de revenus, tant pour les Chinois que pour les Africains (Haifang, 2021).

Au début, cependant, la Chine était rarement la destination préférée des migrants africains. Il offrait plutôt une alternative «deuxième meilleure », généralement obtenue avec l'aide de 'courtiers en migration'. Ces derniers étaient nécessaires parce que les efforts des migrants pour entrer dans l'UE ou aux États-Unis étaient entravés par des régimes d'immigration restrictifs (Haugen, 2012). L'ouverture de l'économie chinoise au début des années 2000 a créé de nouveaux espaces pour les entrepreneurs migrants africains capturant une part des chaînes de valeur internationales, transformant les relations sociales et commerciales, et reconfigurant ainsi l'espace urbain (Lyons \& Brown \& Li, 2008; Kohnert, 2010).

Aux yeux de la plupart des migrants africains, le succès ou la mobilité sociale n'étaient pas évalués par leur vie difficile en Chine, mais par les perspectives d'une future amélioration du niveau de vie chez eux en Afrique (Yin, 2017). Notamment, la Chine du Sud est devenue la nouvelle terre promise pour les migrants africains (Lan, 2017; Marfaing \& Thiel, 2015; Giese \& Marfaing, 2019). Leur satisfaction face à l'amélioration de leur situation économique en Chine, le soutien familial, mais aussi l'expérience antérieure de victimisation dans leur pays d'origine, prédisent fortement leur sentiment de sécurité perçue dans leur pays d'accueil. Leur confiance dans leurs collègues entrepreneurs chinois a également été attribuée à leur évaluation de la sécurité (Song, et al, 2020; Kohnert, 2010).

Cependant, il existait des variations remarquables dans l'évaluation des relations sinoafricaines selon le pays d'origine des migrants africains. Selon une étude de 2008, les $\underline{\text { Kenyans, }}$ les Soudanais et les Éthiopiens étaient les plus positifs à propos des liens ChineAfrique, les Botswanais et les Zambiens les plus négatifs, et les Nigérians, les Ghanéens, les Égyptiens et les Sud-Africains entre les deux. Pourtant, mis à part les Sud-Africains, la

\footnotetext{
${ }^{7}$ Votent contre : République centrafricaine, Tchad, République démocratique du Congo, Dahomey, Gabon, Gambie, Côte d'Ivoire, Lesotho, Libéria, Madagascar, Malawi, Niger, Afrique du Sud, Swaziland, Haute-Volta. Source : Chronology of China-Africa relations.
} 
majorité considérait le développement de la Chine comme un modèle progressiste (Sautmann \& Hairong, 2009).

De plus, il existait une disparité notable entre les dimensions sociales et économiques des réseaux de migrants, une disparité qui était qualifiée de «solidarité limitée » d'une part, et de « confiance exécutoire » d'autre part (Müller \& Wehrhahn, 2011). Les réseaux sociaux n'ont apparemment pas joué un rôle déterminant quant à son avantage économique supposé. La coopération économique entre intermédiaires a plutôt entraîné des inconvénients pour l'entrepreneur individuel. C'était une raison majeure de la stratégie d'action économique hautement autonome des migrants individuels. Néanmoins, les réseaux sociaux étaient essentiels à la survie dans un environnement local hostile et raciste (Müller \& Wehrhahn, 2011).

Les relations des petits commerçants chinois et africains ont fondamentalement changé depuis les manifestations xénophobes contre «les Chinois » dans plusieurs villes africaines au début des années 2000. Cela s'appliquait également à leur incompréhension mutuelle dans les villes chinoises, principalement dans les sous-districts urbains de Guangzhou, Xiaobei et $\underline{\text { Sanyuanli }}$ où des entrepreneurs africains migrants sont arrivés peu de temps après.

Beaucoup d'entre eux, sinon la plupart, ne sont pas venus séjourner de façon permanente dans le pays d'accueil, mais ils ont plutôt agi en tant que résidents, explorant le terrain à la recherche d'un commerce rentable, c'est-à-dire d'exportations chinoises de biens de consommation bon marché vers l'Afrique. Leur intérêt économique mutuel reposait sur le développement de la production industrielle chinoise qui facilitait l'accès des Africains aux produits occidentaux souhaités à un prix abordable (Marfaing \& Thiel, 2015; Giese \& Marfaing, 2019; Kohnert, 2016). Ainsi, les migrants entrepreneurs, qui ont intégré la mondialisation par le bas dans leurs pratiques économiques transnationales, sont devenus euxmêmes porteurs de transformations sociales et économiques dans leurs pays d'accueil respectifs. Le domaine de ces transformations couvrait un éventail remarquable, allant des stratégies d'accès spécifiques aux ressources et à la mobilité sociale, en passant par l'évolution des comportements des consommateurs, les nouvelles normes et pratiques, jusqu'aux modifications des goûts et des modes de vie (Giese \& Marfaing, 2019; Kohnert, 2016).

\section{Problèmes des migrants africains modernes en Chine}

\subsection{Principaux traits et trajectoires de la discussion académique}

Les discussions académiques sur la migration africaine moderne vers la Chine ont souvent pris pour acquis le point de vue et le concept occidental général de la migration transfrontalière. Elle postulait implicitement ou explicitement l'attractivité des bienfaits du développement politique, économique, social et culturel dans les pays hautement industrialisés. En particulier, il mettait l'accent sur les conceptions individualistes des droits de l'homme et embrassait la conviction que l'Occident offrait plus d'opportunités aux migrants de s'intégrer (Nawyn, 2016). De plus, la perspective afro-pessimiste occidentale a favorisé et renforcé les imaginaires préjugés des caractéristiques typiques de «»l'Africain» (par exemple, être paresseux, tardif, peu fiable, etc.) prétendument sans conscience historique et temporelle, comme le montre la controverse sur le discours de Dakar du président français $\underline{\mathrm{N}}$. Sakorzy (Sénégal) en 2007 (McGreal, 2007). Pas étonnant, qu'à ce jour, l'insistance sur la tutelle occidentale et un zèle missionnaire aient été répandus dans le monde occidental, et 
même parmi les Africains, opposés à la cruauté supposée des Chinois vis-à-vis des étrangers (Liang \& Billon, 2020). Pourtant, ces conceptions reflètent également les hiérarchies sociales particulières dans les pays occidentaux d'accueil des migrants, y compris les notions de migration légale et illégale. Cependant, tous ces préjugés entraînent également une vision altérée des opportunités économiques disponibles dans les pays du Sud, avec une fétichisation des économies du Nord (Nawyn, 2016).

Enfin et surtout, le concept occidental de « race», largement partagé par la société chinoise également, a prévalu jusqu'à ce jour, bien que la « race » en tant que catégorie biologique ait été discréditée dans la discussion scientifique il y a longtemps. La pensée chinoise contemporaine est toujours basée sur des concepts de conception de «l'altérité », en se concentrant sur des différences telles que la couleur de la peau, la classe et "l'ethnicité », transmises depuis des siècles (Castillo, 2016).

Quant aux étudiants africains en Chine, qui constituent une part importante de tous les «étrangers » Africaines dans le pays, leurs processus de prise de décision les conduisant à étudier dans les universités chinoises ont été façonnés par des inégalités structurelles. Ainsi, les étudiants ont été contraints de partir à l'étranger pour des raisons politiques et économiques, ou en tant qu'étudiants de la classe moyenne, pas assez aisés selon les normes mondiales, ou en tant des membres de l'élite africaine qui ont profité des réseaux sociaux pour obtenir une bourse diplomatique. Ainsi, les objectifs individuels et familiaux ont été transmis à la migration éducative. Mais la différenciation sociale et la vie sociale quotidienne vécue par les étudiants africains dans le pays d'accueil ont souvent renforcé les préjugés raciaux et les asymétries de développement vis-à-vis des Chinois (Mulvey, 2021; Ho, 2016).

Quant aux petits commerçants, les Africains et les Chinois appartenaient pour la plupart à la classe inférieure. Ils étaient confrontés à la précarité, souvent en raison de leur statut illégal et de leur dépendance à des réseaux douteux et informels, une situation qui s'est encore accentuée au milieu de la pandémie de COVID-19 (Asiedu, 2020; Jordan et al, 2021).

Cependant, les forces structurantes de la précarité, provoquées par des facteurs de pauvreté mondiale et établies par des politiques et institutions nationales et locales opposées, ont également généré des défis et des opportunités pour les familles mixtes afro-chinoises de mobilités superposées (Jordan et al, 2021). Un exemple est la solidarité remarquable au sein des réseaux de migrants africains en Chine concernant les soins de santé. Les Africains n'avaient qu'un accès restreint aux services de santé locaux en raison d'une combinaison d'obstacles sociaux et structurels. Ainsi, près de 5000 étudiants africains à $\underline{\text { Whan }}$, le lieu du premier confinement de la pandémie de Corona en janvier 2020, manquaient d'argent et de nourriture pendant le confinement de Corona (Bodomo, 2020). Pourtant, par des initiatives stratégiques menées par la communauté, de nombreuses communautés africaines ont essayé de surmonter les restrictions. Ainsi, la communauté ghanéenne de Guangzhou a fait un don en espèces et a organisé des soins de santé pour sa communauté. D'autres communautés ont également compilé des informations sur les visites des prestataires de soins de santé d'Afrique pour leurs membres et encouragent ces spécialistes invités à consulter de manière informelle et volontaire les nécessiteux. Les anciens de la famille et les chefs religieux ont joué un rôle majeur en tant que prestataires de soins de santé (Bodomo, 2020).

À l'avenir, les enfants de parents africains des petits commerçants, qui sont nés et ont grandi en Chine, pourraient également servir de lien entre les deux régions. Ces perspectives ont été grandement facilitées par l'accueil et la logistique d'infrastructures informelles mises en place par les migrants africains à la fin des années 1990, qui ont par la suite évolué et se sont 
adaptées. Ainsi, la mobilité et les séjours individuels et collectifs joueront un rôle de plus en plus important dans la gouvernance des relations commerciales par le courtage culturel et économique (Haugen, 2019). Cette infrastructure sociale spécifique a facilité la mobilité des personnes et des biens et a augmenté le rythme de circulation des capitaux commerciaux (Haugen, 2019; Cheuk, 2021). Cependant, le flux migratoire souvent décrit et simplifié à l'extrême comme basé sur des facteurs d'attraction et de répulsion bipolaires, correspondait plutôt à un concept de trajectoires migratoires circulaires, transitoires et conceptionnelles (Marfaing \& Thiel, 2015; Giese \& Marfaing, 2019; Cheuk, 2019; Carling \& Haugen, 2021). Par conséquent, le concept de «l'état transitoire » serait un terme plus approprié. Cela vaut non seulement pour les Africains vivant en Chine, mais aussi pour les Chinois en Afrique, à la fois souvent sans désir de s'intégrer dans le pays d'accueil, et fréquemment aussi sans possibilité d'intégration, ce qui rendait leur vie instable et leur avenir incertain (Leslie, 2018).

Déjà, la loi chinoise sur les migrations de 2013 avait souligné la distinction entre immigrés et citoyens nés dans le pays. La loi, appelée «Loi sur l'administration des sorties et des entrées » (Exit and Entry Administration Law, EEAL), s'est particulièrement concentrée sur l'entrée, le séjour et l'emploi illégaux (Cissé, 2021). Le profilage racial des Africains par la police chinoise faisait des Africains des cibles particulièrement vulnérables pour les policiers, qui subissaient de fortes pressions pour améliorer leurs statistiques de performance et étaient souvent également motivés par des incitations monétaires. Le Bureau de la sécurité publique de Guangzhou, par exemple, a classé les Africains de la ville comme «triple illégaux », ou sanfei 三非, une expression faisant référence à ceux qui entrent, séjournent et travaillent illégalement en Chine (Huang, 2019).

En réponse, plusieurs migrants africains ont développé des stratégies d'adaptation adaptées pour contrer la stigmatisation de l'illégalité, compte tenu de la politique d'immigration stricte de Pékin, en utilisant différents niveaux d'interactions avec la société locale. À Guangzhou par exemple, les migrants africains ont déjoué l'administration en collaborant avec des partenaires commerciaux chinois des régions moins développées du pays. La collaboration avec ces migrants a permis aux commerçants africains de contourner certaines des contraintes imposées par la loi sur l'immigration de l'État. Il a également étendu leurs options de mobilité au-delà de Guangzhou à d'autres villes chinoises comme Yiwu (Lan, 2016).

De plus, les motivations de l'administration derrière le projet de rénovation urbaine dans le quartier de Xiaobei à Guangzhou, dans le cadre de la vision du gouvernement municipal de construire une ville «propre, sûre et ordonnée », affectaient manifestement les populations marginalisées de manière disproportionnée. Ceux-ci comprenaient non seulement les résidents transnationaux, mais également les migrants nationaux Chinois, dans le cadre des efforts visant à contrôler la «population flottante» de migrants nationaux (Han et non-Han) (Wilczak, 2017). Néanmoins, les Africains étaient les plus ciblés (Africans in Guangzhou, Wikipedia). La stigmatisation des migrants africains à la suite de la pandémie d'Ebola, qui a sévi en Afrique de l'Ouest en 2014 et 2015 par exemple, a encore accru la difficulté d'accéder aux informations et aux services de santé et les a par la suite rendus plus vulnérables à la discrimination (Lin, 2015).

Ainsi, la migration pourrait à la fois enrichir et mettre à rude épreuve le commerce bilatéral et les relations étrangères, et même avoir un impact sur la stabilité sociale des pays d'accueil. Le recours des migrants africains aux mariages mixtes comme stratégie d'adaptation et l'engagement de quelques migrants dans le trafic de drogue, la fraude sur Internet et la prostitution pourraient mettre en danger les relations de la Chine avec l'Afrique (Elochukwu, 2016). Pékin a dû faire face au dilemme entre le discours d'amitié sino-africain au niveau de 
la politique étrangère et la montée de l'afrophobie au niveau local. Elle considérait ses alliés africains principalement comme des partenaires politiques stratégiques et non comme des partenaires économiques sur un pied d'égalité. Par conséquent, la propagande chinoise s'est concentrée sur l'aide de la Chine à l'Afrique, et non sur la contribution de cette dernière à l'économie chinoise. Cela a également perpétué les stéréotypes négatifs des Chinois selon lesquels les Africains sont pauvres et nécessiteux (Yin, 2017).

En bref, le tissu social du pays d'accueil du migrant incarne en grande partie des traits majeurs d'exclusion des «étrangers ». Ces derniers se concentrent souvent sur l'ethnicité, la race, la

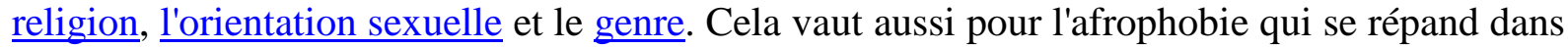
la Chine contemporaine. Ainsi, l'actualité s'est focalisée sur l'expulsion des migrants africains d'appartements et d'hôtels en Chine. En fait, on estime que 500000 Africains vivent en Chine. La pandémie de Corona a aggravé leur situation.

\subsection{Migration africaine vers la Chine depuis 1960, déclenchée par l'évolution des exportations chinoises de biens de consommation vers l'Afrique}

Graphique 3 : migrants africains réguliers en Chine, 1960 - 2000

(total de 45 pays africains, en milliers, chiffres officiels)

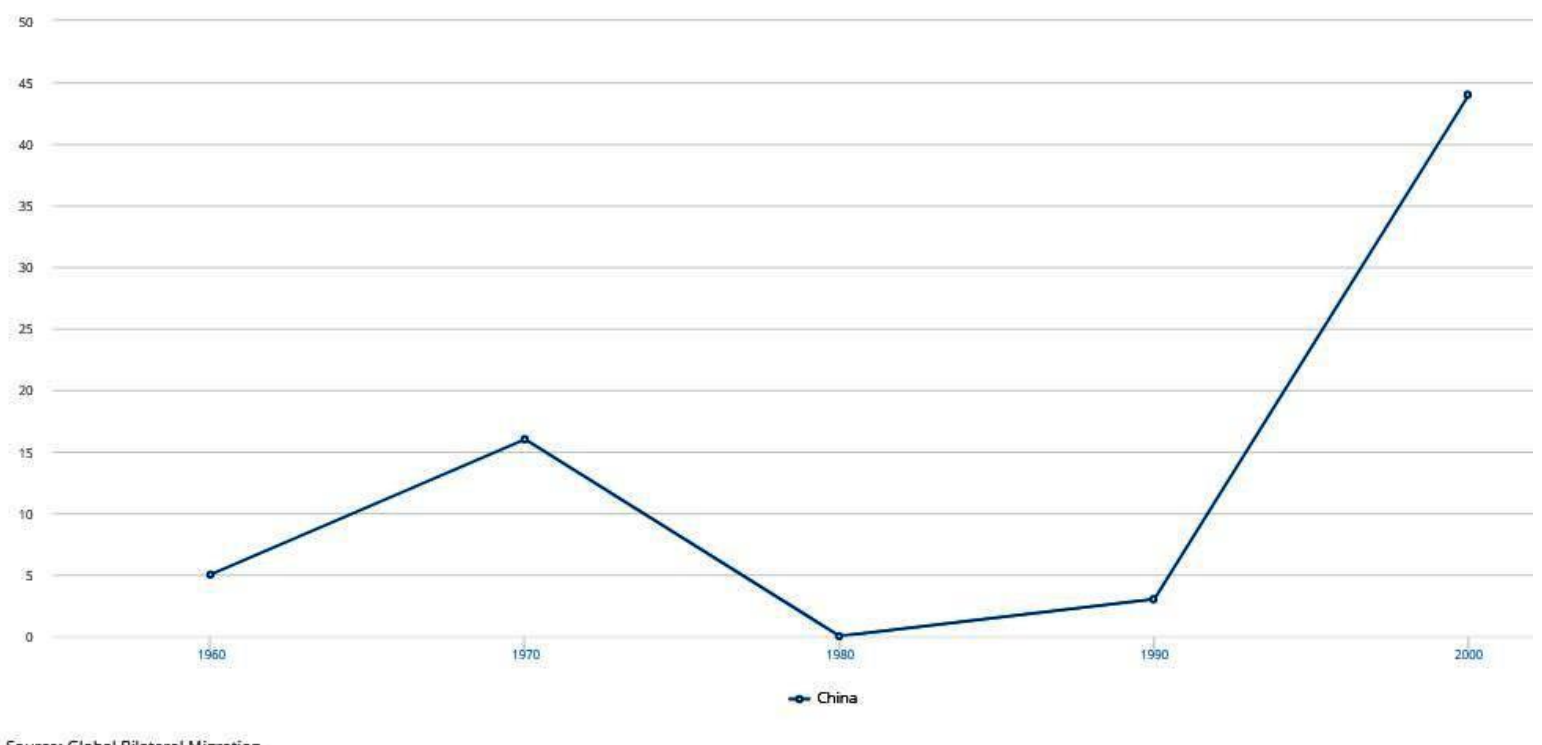

Source: WB (2021): Databank - Global Bilateral Migration

(Dernière mise à jour : 28/06/2011, aucune donnée plus récente disponible)

L'afrophobie croissante était probablement également due à la migration croissante d'Africains vers la Chine au cours des dernières décennies. Selon les chiffres officiels, le total des migrants africains réguliers en Chine n'a augmenté que modestement, passant de 5000 à 45000 au cours de la période 1960 à 2000 (voir graphique 3). Ils pourraient au mieux refléter la direction de l'augmentation, et non les chiffres factuels. Malheureusement, les données officielles les plus récentes pour les deux dernières décennies ne sont pas disponibles. Selon des bonnes estimations, cependant, rapportées dans la presse chinoise et occidentale, diverses évaluations ont circulé sur les migrants africains réguliers et irréguliers en Chine, qui étaient toujours beaucoup plus élevées, allant de 100000 à 200000 déjà en 2008 (Gazibo \& 
Alexeeva, 2012). Environ une décennie plus tard, il y avait environ 500000 migrants africains réguliers et irréguliers vivant en Chine en 2020, selon des estimations bien informées.

Faute de données fiables, le deuxième meilleur indicateur des tendances à la hausse de l'immigration pourrait être la croissance du commerce et des investissements sino-africains. Depuis le début des années 2000, un nombre croissant de petits commerçants africains ont tenté d'obtenir leur part du gâteau, parallèlement et accompagnant la montée du commerce et des investissements chinois en Afrique, en organisant l'importation de marchandises chinoises directement depuis ses sources en Chine (Marfaing \& Thiel, 2015; Giese \& Marfaing, 2019; Kohnert, 2016).

Le commerce Chine-Afrique n'a commencé à augmenter sensiblement qu'au début de 2000 (voir graphique 6). Le volume des échanges a augmenté régulièrement au cours des 16 dernières années. En 2019, la Chine est devenue le plus grand partenaire commercial bilatéral de l'Afrique avec un commerce total de US\$ 200 milliards (Stein \& Uddhammar, 2021). En 2019, les exportations chinoises vers l'Afrique se sont élevées à US\$ 113 milliards et les importations en provenance d'Afrique ont atteint US\$ 78 milliards. La plupart de ces échanges se faisaient avec les pays subsahariens qui représentaient les deux tiers des échanges. Cependant, sa répartition régionale était inégale, les six principales destinations d'exportation du commerce chinois avec l'Afrique, parmi lesquels l'Afrique du Sud, le Nigéria et l'Égypte ont absorbé plus de la moitié des exportations totales. Il en va de même pour les importations chinoises en provenance d'Afrique. Six pays représentaient $68 \%$ des importations totales, le pétrole angolais représentant près d'un tiers (Stein \& Uddhammar, 2021).

\section{Graphique 4 : Termes de l'échange de l'Afrique du Sud, $2000-2020$ (OCDE) ${ }^{8}$}

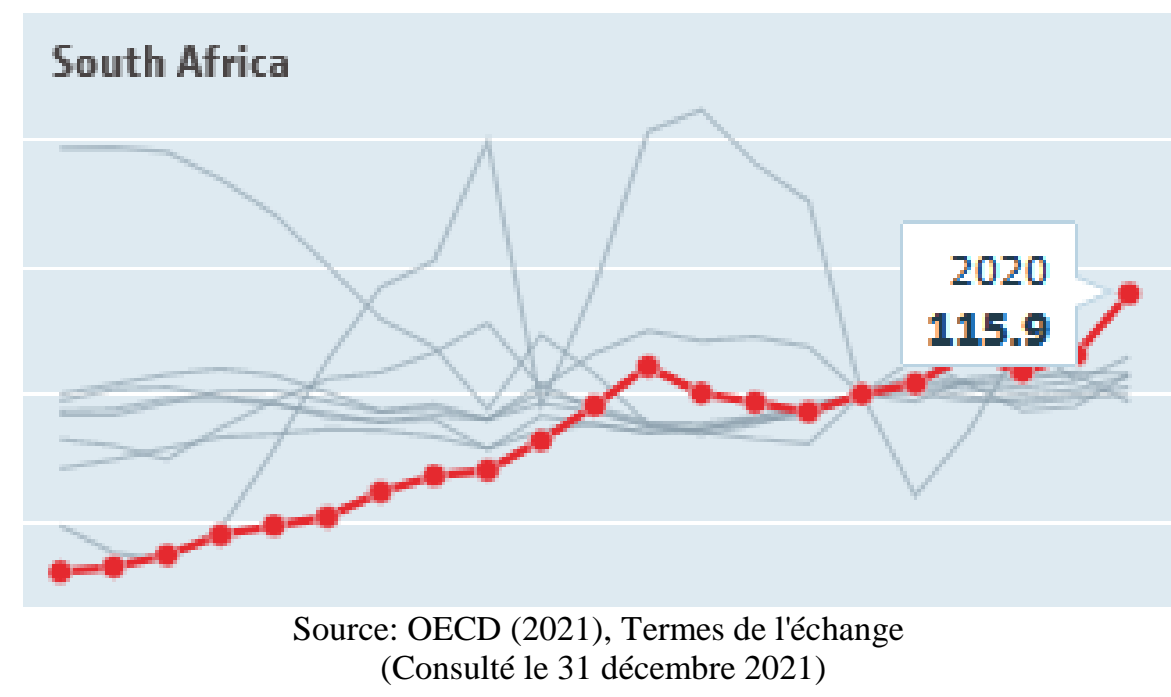

L'augmentation du commerce bilatéral a abouti à une situation gagnant-gagnant pour tous les partenaires, du moins selon la plupart des gouvernements impliqués des deux côtés. Le fait est que pour les pays africains, ce fut un stimulus majeur de la croissance économique considérable de la fin des années 1990 à 2014.

\footnotetext{
${ }^{8}$ «Les termes de l'échange sont définis comme le rapport entre l'indice des prix à l'exportation et l'indice des prix à l'importation. Si les prix à l'exportation augmentent plus que les prix à l'importation, un pays a des termes de l'échange positifs, car pour le même montant d'exportations, il peut acheter plus d'importations ». (OECD, 2021):
} 
Graphique 5 : Développement projeté de l'économie de la téléphonie mobile en ASS, 2020-2025

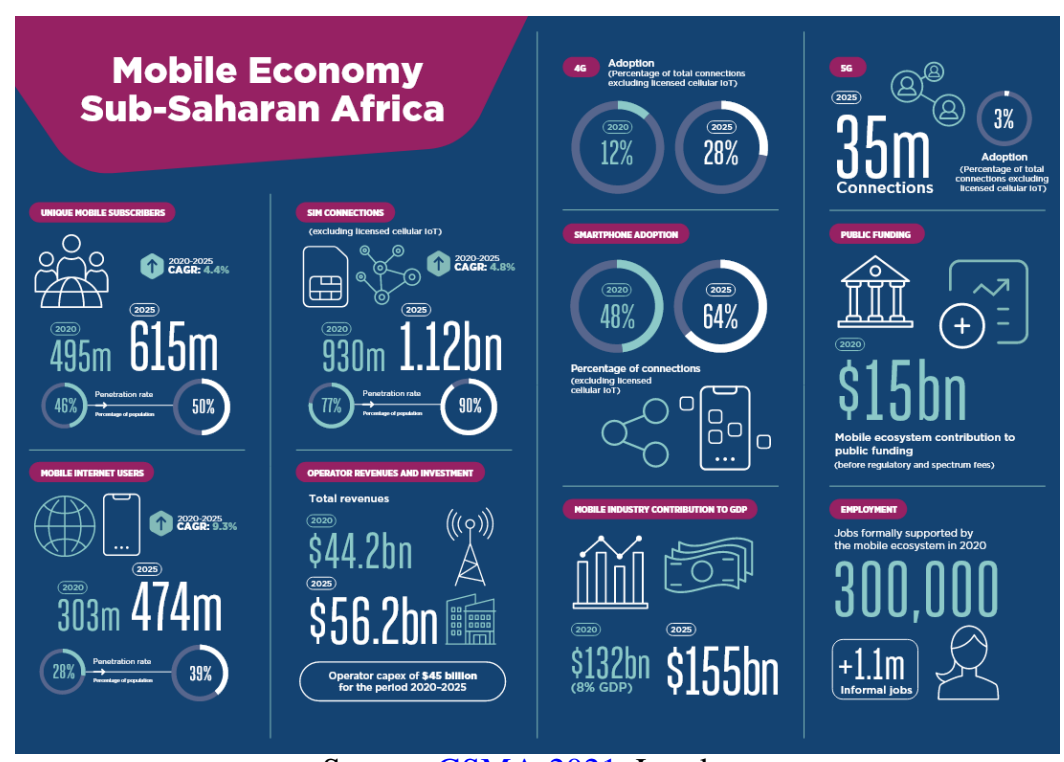

Source: GSMA-2021, London:

Global System for Mobile Communications, GSMA

La Chine a fourni aux Africains des biens de consommation bon marché, abordables également pour les Africains pauvres, ainsi que des biens d'équipement. De plus, les biens de consommation avancés, comme les téléphones portables, désormais abordables également pour les citoyens ordinaires, ont contribué à réduire le fossé numérique avec les pays développés, et à créer de nouveaux marchés et la prise de pouvoir des défavorisées.

En outre, la demande croissante de la Chine pour les ressources africaines a amélioré les termes de l'échange de l'Afrique ainsi que ses revenus financiers. Elle a permis aux économies africaines de diversifier leur commerce extérieur, notamment dans les économies les plus avancées d'Afrique subsaharienne comme l'Afrique du Sud (voir graphique 4).

Depuis les années 2010, un déplacement des principaux investisseurs des investissements directs à l'étranger (IDE) en Afrique, des pays occidentaux traditionnels fortement industrialisés vers les pays BRICS (Brésil, Russie, Inde, Chine, Afrique du Sud) a été observé. La Chine et l'Inde sont devenues des acteurs mondiaux importants en Afrique, rivalisant pour l'espace stratégique (Chakrabarti et Ghosh, 2014). Alors que l'ambition de Pékin était principalement liée à la poursuite de la politique étrangère «Une seule Chine », New Delhi visait à contrebalancer l'influence chinoise dominante en Afrique et à obtenir le soutien de son agenda politique concernant le commerce agricole, le changement climatique et l'adhésion permanente au Conseil de sécurité des Nations unies (Chakrabarti, \& Ghosh, 2014).

Selon le Rapport sur les investissements dans le monde de la CNUCED pour 2020, les investissements directs à l'étranger (IDE) en Afrique devaient chuter de $25 \%$ à $40 \%$ en 2020, première année de la pandémie de Corona. La tendance négative serait aggravée par la faiblesse des prix des matières premières. Déjà avant la pandémie, en 2019, les flux d'IDE vers l'Afrique avaient déjà diminué de $10 \%$ à US\$ 45 milliards (UNCTAD, 2020). 
Graphique 6 : Croissance des échanges de biens et services de la Chine avec l'Afrique, 1992-2019 (US\$ milliards, prix courants)

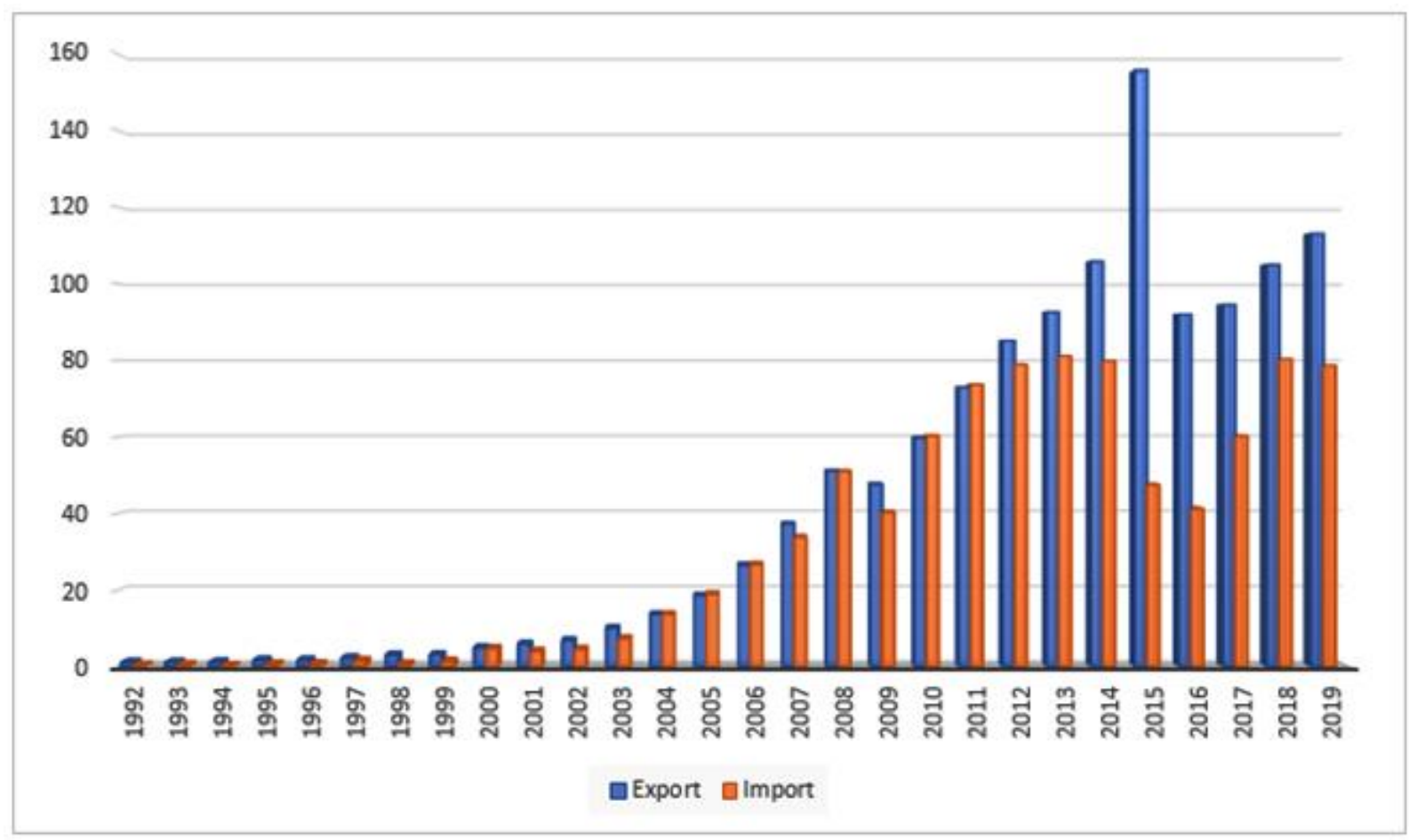

Source: Stein \& Uddhammar, 2021

UN Comtrade and the SAIS China Africa Research Initiative (SAIS-CARI)

Quant aux IDE chinois, ils ont été principalement affectés à la consolidation et à l'extension des infrastructures de l'Afrique. Ces derniers étaient pour la plupart dépassés depuis l'époque coloniale, comme les chemins de fer, les routes, les ports, les aéroports et les égouts, mais aussi des projets «politiques » prestigieux ont été financés, comme des stades, des bâtiments parlementaires et des résidences pour les chefs d'État. En outre, la Chine a fourni à l'Afrique un savoir-faire et une technologie modernes et les investissements chinois ont augmenté la capacité de production globale des économies africaines (Stein \& Uddhammar, 2021).

Graphique 7 : Les plus grands marchés d'exportation de la Chine en Afrique (2019)

\begin{tabular}{|l|c|c|}
\multicolumn{1}{c|}{ Country } & $\begin{array}{c}\text { Exports, in USD } \\
\text { billion }\end{array}$ & $\begin{array}{c}\text { \% of Chinese exports to } \\
\text { Africa }\end{array}$ \\
\hline South Africa & 16.6 & $14.6 \%$ \\
\hline Nigeria & 16.6 & $14.6 \%$ \\
\hline Egypt & 12.2 & $10.7 \%$ \\
\hline Algeria & 6.9 & $6.1 \%$ \\
\hline Kenya & 4.9 & $4.3 \%$ \\
\hline Ghana & 4.9 & $4.3 \%$ \\
\hline Total six countries & & $\mathbf{5 4 . 6 \%}$
\end{tabular}

Source: China-Africa Research Initiative ${ }^{6}$

Source: Stein \& Uddhammar, 2021

Cependant, la conquête chinoise des marchés africains avait aussi ses inconvénients. Ainsi, il a entravé l'émergence d'un secteur manufacturier africain naissant, par exemple dans l'industrie textile (Sylvanus, 2013), déplacé les entreprises et les travailleurs locaux, enfin et 
surtout, en raison des salaires de dumping souvent injustes pour les travailleurs chinois sur les chantiers de construction dans divers pays africains et des conditions de travail déplorables pour les travailleurs africains dans les entreprises chinoises (Xiaoyang, 2016; Mbamalu, 2018). En outre, les exportations chinoises vers l'Afrique pourraient évincer les exportations des économies africaines les plus avancées, par exemple l'Afrique du Sud.

Graphique 8 : Principales destinations d'importation de la Chine en Afrique (2019)

\begin{tabular}{|l|c|c|}
\multicolumn{1}{c|}{ Country } & $\begin{array}{c}\text { Imports, in USD } \\
\text { billion }\end{array}$ & $\begin{array}{c}\text { Percent of Chinese } \\
\text { imports from Africa }\end{array}$ \\
\hline Angola & 23.3 & $29.8 \%$ \\
\hline South Africa & 9.5 & $12.1 \%$ \\
\hline Republic of Congo & 5.9 & $7.5 \%$ \\
\hline DRC & 4.9 & $6.2 \%$ \\
\hline Libya & 4.7 & $6.0 \%$ \\
\hline Gabon & 4.6 & $5.9 \%$ \\
\hline Total six countries & & $\mathbf{6 7 . 5 \%}$ \\
\hline
\end{tabular}

Source: China-Africa Research Initiative ${ }^{7}$

Source: Stein \& Uddhammar, 2021

Graphique 9 : Principaux destinataires des IDE chinois (2019, en milliards USD)

\begin{tabular}{|l|c|c|}
\multicolumn{1}{c|}{ Country } & $\begin{array}{c}\text { Total stock, in } \\
\text { USD billion }\end{array}$ & $\begin{array}{c}\text { Percent of Chinese FDI in } \\
\text { Africa }\end{array}$ \\
\hline South Africa & 6.1 & $13.8 \%$ \\
\hline DRC & 5.5 & $12.5 \%$ \\
\hline Angola & 2.9 & $6.5 \%$ \\
\hline Zambia & 2.8 & $6.5 \%$ \\
\hline Ethiopia & 2.5 & $5.6 \%$ \\
\hline Ghana & 1.8 & $4.1 \%$ \\
\hline Total six countries & $\mathbf{2 1 . 6}$ & $\mathbf{4 9 . 1 \%}$ \\
\hline
\end{tabular}

Sources: UNCTAD and China-Africa Research Initiative ${ }^{d}$

Source: Stein \& Uddhammar, 2021

Tout cela peut créer de nouvelles dépendances des pays africains avec des implications stratégiques, et de nombreux politiciens africains accusent Pékin de comportement néocolonial (Lisimba, 2020). Par conséquent, la question reste ouverte de savoir si la politique de commerce extérieur de la Chine intègre un modèle chinois spécifique de développement à reproduire en Afrique (Stein \& Uddhammar, 2021).

La Chine a créé 25 zones économique exclusive (ZEE) dans 16 pays africains, enregistrées auprès du ministère chinois du Commerce. Ils visaient à créer une plate-forme de la stratégie d'engagement de Pékin en Afrique comme «mutuellement bénéfique, basée sur des décisions basées sur le marché et des investissements par des entreprises chinoises, combinées avec le soutien et les subventions du gouvernement chinois (Bräutigam \& Xiaoyang 2011). À première vue, les ZEE semblaient promouvoir le modèle de Pékin « Flying Geese » («Oies volantes ») ou «Leading Dragon » («Dragon principal ») pour un développement industriel africain soutenu. Cependant, l'expérience de l'Afrique avec les ZEE a été décevante. Ils n'ont 
pas réussi à attirer suffisamment d'investissements, à promouvoir les exportations ou à créer un développement industriel durable. Les ZEE étaient trop axées sur les gains à court terme et ne pouvaient pas atténuer la médiocrité des infrastructures, les conflits d'intérêts entre les gouvernements hôtes et les investisseurs, les conflits sociaux et politiques nationaux et l'instabilité politique, ni régler les problèmes environnementaux (Okereby, 2019).

Pékin a décidé que ses propres entreprises prendraient l'initiative de développer ces zones. Cependant, des rapports critiques ont insisté sur le fait que ces zones étaient plutôt destinées à aider la propre restructuration de la Chine, permettant aux industries à forte intensité de maind'œuvre et moins compétitives, telles que le textile, la maroquinerie et les matériaux de construction, de se déplacer vers l'étranger. Pourtant, ce sont surtout les gouvernements africains eux-mêmes qui sont responsables de l'échec des ZEE. Ses dirigeants politiques manquaient de détermination pour intégrer ces zones dans la politique industrielle globale et s'en servaient souvent pour leur propre avancement politique. La plupart du temps, les fabricants locaux n'étaient pas autorisés à opérer dans ces zones, même lorsque les développeurs chinois et les gouvernements hôtes partageaient conjointement la propriété. Ainsi, le gouvernement de l'État de Lagos détenait $40 \%$ des parts de la zone de libre-échange de Lekki au Nigeria, mais les fabricants locaux ne pouvaient pas l'utiliser, car les ZEE étaient bouclées comme une base militaire (Okereby, 2019).

Néanmoins, la Chine a continué à investir massivement à travers le continent tout au long de la pandémie de COVID-19 (IISD, 2021). C'est actuellement le quatrième investisseur en Afrique. Ces derniers temps, Pékin a progressivement investi dans le cadre de son ambitieuse «Initiative Ceinture et Route » dans le secteur des services africains, y compris des soussecteurs tels que la recherche scientifique et les services technologiques, les transports, l'entreposage et les services postaux. Cependant, un nombre croissant d'États africains ont dû suspendre ou abandonner les projets ces derniers temps parce qu'ils n'étaient pas en mesure de rembourser leurs prêts (IISD, 2021).

\subsection{La baisse du flux des envois de fonds pendant la pandémie de Corona}

La pandémie de Covid-19 depuis janvier 2020 a encore accru le risque et la précarité de la vie quotidienne des migrants africains en Chine, notamment celle des petits commerçants en situation irrégulière. Le verrouillage a augmenté leur risque d'interception par les autorités de l'État chaque fois qu'ils ont quitté leur domicile et visité des sites de marché pour leur entreprise. Ils dépendaient souvent des services offerts par des agents de confiance, habituellement d'autres migrants eux-mêmes, par l'intermédiaire desquels ils transféraient et recevaient leurs biens commerciaux et leurs fonds (Gill, 2020). Cela a également eu un impact sur leurs familles à la maison. 
Graphique 10 : Pas d'accès internet, pas de compte bancaire, parmi ces Africains dépendants des envois de fonds

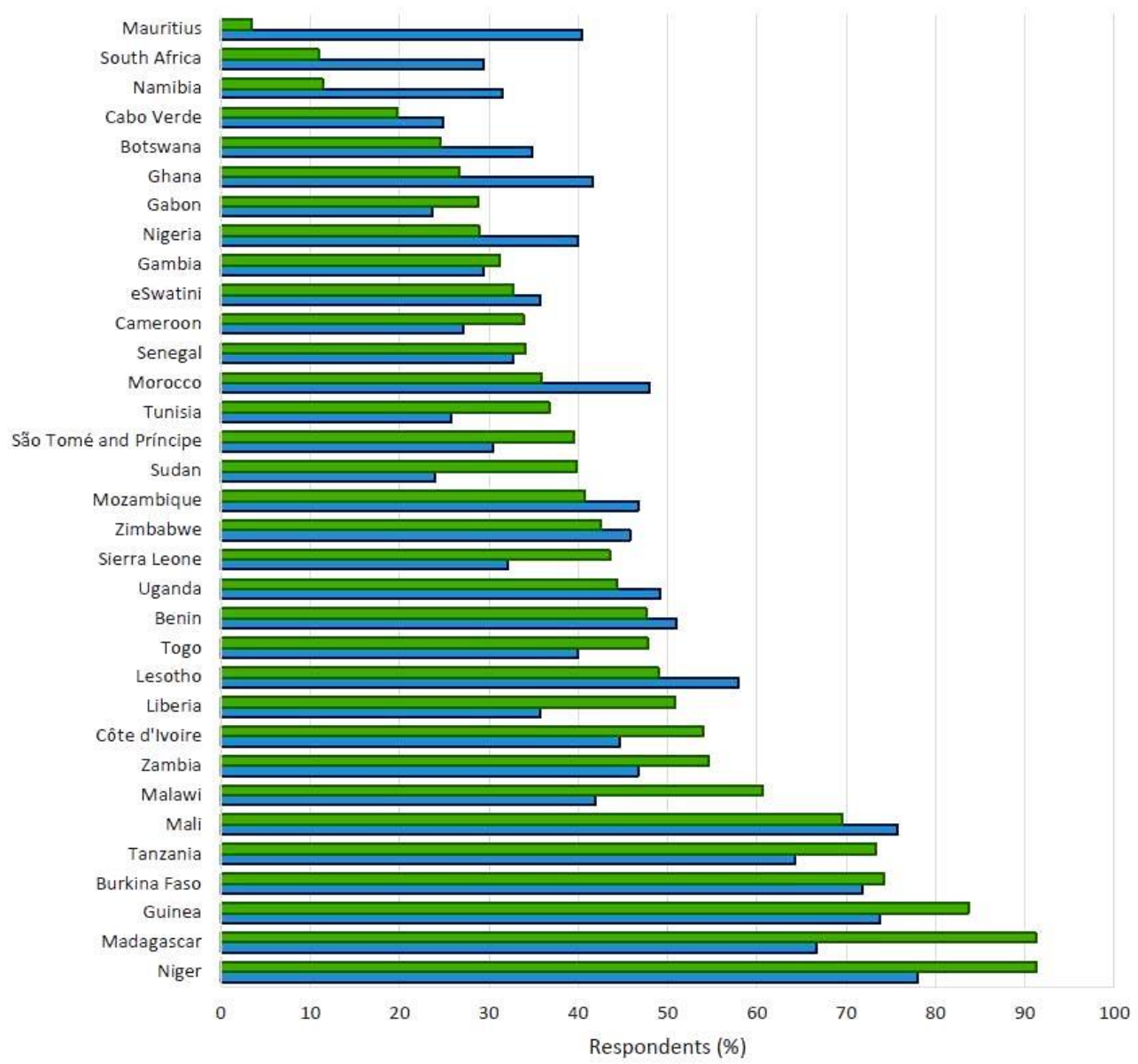

Source: Kalantaryan, S \& McMahon, S (2020)

La Banque mondiale a prévu une baisse sans précédent des envois de fonds pour 2020 et 2021 à l'échelle mondiale. Les envois de fonds mondiaux pourraient chuter de 19,9\% et affluer vers l'Afrique même de 23,1 \% en 2020. Cela aurait supprimé une source majeure de revenus pour de nombreuses familles africaines restées au pays et aurait affecté leur capacité à répondre à la crise et à s'en remettre (Kalantaryan, \& McMahon , 2020). En 2021, la Banque mondiale a ajusté ses chiffres à une baisse des envois de fonds de $12,5 \%$ pour l'Afrique subsaharienne en 2020, qui était presque entièrement due à une baisse de $28 \%$ des envois de fonds vers le Nigéria. Hors de Nigeria, les envois de fonds vers l'ASS ont même augmenté de $2,3 \%$ en moyenne. La croissance des envois de fonds a augmenté en particulier pour la Zambie (37 \%), le Mozambique (16\%), le Kenya (9 \%) et le Ghana (5\%). En 2021, les envois de fonds vers la région avaient été projetés par la BM en hausse de 2,6\% en moyenne, soutenus par l'amélioration des perspectives de croissance dans les pays à revenu élevé (Kalantaryan \& McMahon, 2020). Malheureusement, une ventilation des données générales sur les envois de fonds des migrants africains en Chine n'est pas encore disponible. Les premières études de cas (voir ci-dessous), cependant, suggèrent qu'il pourrait être considérable.

Les fermetures de banques et de bureaux de Western Union, ces derniers utilisés surtout par les Africains les plus pauvres qui n'avaient pas de compte bancaire pour les transferts d'argent, ainsi que la fermeture d'autres opérateurs de transfert en Afrique et en Chine, ont encore 
aggravé le problème. En dernier ressort, un passage aux transferts de fonds numériques aurait été possible, mais uniquement pour ceux qui disposaient de téléphone portable et d'accès Internet plus sophistiqués. Pourtant, selon une étude récente d'Afrobaromètre dans huit pays (Bénin, Lesotho, Tanzanie, Madagascar, Burkina Faso, Guinée, Mali, Niger), plus de la moitié des personnes qui dépendaient des envois de fonds n'avaient pas accès à Internet via un téléphone mobile (Kalantaryan, \& McMahon, 2020).

\section{Implications de l'afrophobie sur la position de la Chine dans la compétition mondiale pour les ressources et les marchés africains}

La migration africaine vers la Chine a été déclenchée par l'évolution des exportations et des investissements chinois vers l'Afrique et l'afflux concomitant de travailleurs et de petits commerçants chinois. Les entreprises de construction chinoises ont souvent insisté pour amener leurs collègues chinois à effectuer la plupart des opérations du projet (Dalibi \& Bello, 2017). De plus, un nombre croissant d'étudiants africains tentaient d'obtenir leurs diplômes dans des universités chinoises. L'afrophobie croissante a été provoquée par la migration croissante des Africains vers la Chine au cours des dernières décennies, à côté de la prévalence générale d'attitudes racistes latentes parmi la population chinoise, comme décrit cidessus. Les gouvernements africains, l'Union africaine (UA) et les États-Unis ont protesté avec véhémence et tenté de faire pression sur Pékin pour qu'il mette fin à ces attentats, mais surtout en vain (Asiedu, 2020).

La réputation négative de la Chine en raison de l'afrophobie croissante pourrait c. p. déclencher un déplacement de la demande africaine de commerce et d'investissement vers des fournisseurs mondiaux compétitifs comme l'Inde, l'UE et les États-Unis. Cela pourrait donc avoir un impact significatif sur la position de Pékin dans la compétition mondiale pour les ressources et les marchés africains. Néanmoins, il faut toujours garder à l'esprit que l'opposition locale à la migration et aux migrants est courante globalement, y compris dans de nombreux pays de l'UE et aux États-Unis.

Depuis les années 2000, il y avait des inquiétudes croissantes concernant la braderie et «l'accaparement des terres» en Afrique. Les médias occidentaux ainsi que les études universitaires l'ont attribué principalement à la cupidité de la Chine et des États arabes du Golfe. En outre, des acteurs mondiaux concurrents, notamment les États-Unis, l'UE et l'Inde, ont saisi l'occasion pour blâmer le «nouvel impérialisme » chinois pour d'autres méfaits. En premier lieu figurait la «ruée » impitoyable pour les ressources de l'Afrique, au cours de laquelle les acteurs financiers et étatiques chinois auraient adapté les terres communales et privatisées sous leur contrôle dans le contexte de la volatilité mondiale des prix des matières premières ou des super-cycles économiques transnationaux, et des luttes entre les monopoles et les États sur les ressources africaines (Aj1, 2010). Tout cela a renforcé la sinophobie en Afrique.

Pourtant, là encore, il fallait admettre que les investissements de Pékin en Afrique avec son orientation à long terme vers des flux de matières premières stables et la construction d'une infrastructure à l'échelle du continent, se comparaient positivement, par ex. avec l'orientation américaine vers l'accumulation et les profits à court terme. Cette dynamique peut également avoir contribué à stabiliser la demande de matières premières et donc des politiques de développement africaines qui reposent encore fortement sur la stabilité de la demande et des prix des matières premières (Ajl, 2010). 
Cependant, les visions gauchistes d'un changement de régime, permises par le mouvement des non-alignés, notamment les pays BRICS mentionnés ci-dessus, pourraient obscurcir le «nouvel-impérialisme » sous le déguisement de la caractérisation de la Chine comme l'acteur anticapitaliste le plus important à l'échelle mondiale. Apparemment, le passage du modèle colonial de production de cultures de rente orientée vers l'exportation, envisagé par la Conférence de Bandung en 1955, vers une société plus équitable par la nationalisation des terres, et les tentatives de création d'une plus-value durable au cours de la libération nationale, n'a pas réalisé. En effet, aussi les nouveaux acteurs asiatiques, Chine et Inde, ont contribué à l'accélération de la paupérisation rurale et à la différenciation sociale agraire (Ajl, 2010; Mupambwa \& Xaba 2021).

Un mouvement politique actuel, particulièrement prôné dans les anciennes puissances coloniales, et façonné par un nationalisme conservateur de droite, embrassant le racisme, offre une fois de plus une justification de l'effort colonial. Cependant, le préjugé selon lequel le colonialisme, malgré ses multiples erreurs, avait inspiré le développement dans les sociétés africaines qui ont par la suite suivi le trait de «modernisation », y compris la démocratie, une gouvernance éclairée et une administration efficace, qui à son tour a inspiré la conscience nationale et les luttes de libération nationale, a subi de dures critiques académiques (Parashar \& Schulz, 2021). À cet égard, il est tout à fait compréhensible que de nombreux Africains aient considéré l'engagement de Pékin en Afrique comme un soulagement par rapport à la « mission civilisatrice» centenaire des anciennes puissances coloniales. Par conséquent, l'aide chinoise, ses prêts bonifiés et non-conditionnels et son engagement à ne pas s'ingérer dans les affaires intérieures africaines ont été les bienvenus. De plus, l'engagement de la Chine correspondait également au mécontentement croissant des Africains à l'égard de l'approche occidentale, y compris son héritage historique, sa présence militaire post-coloniale en Afrique et son impérialisme culturel à travers l'imposition de valeurs et de normes occidentales (Galchu, 2018 )

Dernièrement, cependant, les relations sino-africaines se sont refroidies. L'influence croissante de Pékin, associée à ses activités mercantilistes perçues comme égoïstes et intraitables, a conduit à un ressentiment croissant. De plus, la négligence chinoise des principes universels des droits de l'homme, y compris l'observation des meilleures pratiques mondiales en matière de gestion du travail et de l'environnement dans les pays africains, a fait que les relations chinoises avec l'Afrique sont au banc d'essai (Okon \& Ojakorotu, 2021). Cela a été intensifié par les politiques discriminatoires envers les Africains en Chine qui ont déclenché des tensions diplomatiques (Sibiri, 2021). En Afrique et ailleurs, l'Initiative de la

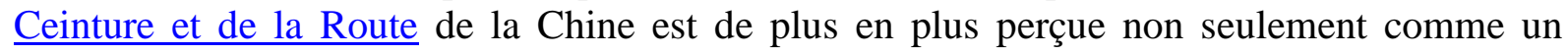
gigantesque projet d'infrastructure et de télécommunications, mais aussi comme une expression de l'hégémonie mondiale chinoise du XXIe siècle. Pourtant, tout positionnement crédible des États-Unis contre le racisme chinois exigerait que la société américaine ellemême combatte efficacement le racisme dans ses propres rangs (Dever \& Dever, 2021).

Ainsi, la migration pourrait à la fois enrichir et mettre à rude épreuve le commerce bilatéral et les relations étrangères, et même avoir un impact sur la stabilité sociale des pays d'accueil. Pékin a dû faire face au dilemme entre le discours d'amitié sino-africain au niveau de la politique étrangère et la montée de l'afrophobie au niveau local. Il a propagé un partenariat sur un pied d'égalité avec l'Afrique, apparemment bâti sur du sable. Le gouvernement chinois voyait ses alliés africains, dans des manœuvres tactiques à peine voilées, principalement comme des homologues politiques stratégiques et non comme des partenaires économiques à armes égales. Au lieu de cela, la propagande chinoise s'est concentrée sur l'aide de la Chine à 
l'Afrique, et non sur la contribution de cette dernière à l'économie chinoise, sans parler de la course mondiale aux ressources et aux marchés africains. Cela a également perpétué les stéréotypes négatifs chinois des Africains comme pauvres et nécessiteux. Bref, le tissu social du pays d'accueil du migrant incarne en grande partie des traits majeurs d'exclusion des «étrangers ». La pandémie de Corona a aggravé leur situation.

\section{Conclusion}

Selon les médias occidentaux, la Chine représentait principalement des clichés négatifs dans la course mondiale aux ressources et aux marchés de l'Afrique. Il représentait l'oppression, la centralisation et une économie de gouvernement central. Son principal concurrent asiatique, l'Inde, a au contraire été salué comme un phare de la démocratie en Asie par rapport à la Chine (s. Kohnert, 2021). Notamment, pendant la guerre froide qui a propagé un « choc des systèmes » ou même un « choc des civilisations » (S.P. Huntington), l'Inde a été soutenue en tant que «plus grande démocratie du monde ». Il défendait la liberté individuelle, la décentralisation et la concurrence.

Graphique 11 : Satisfaction des citoyens

vis-à-vis de la performance du gouvernement dans six pays

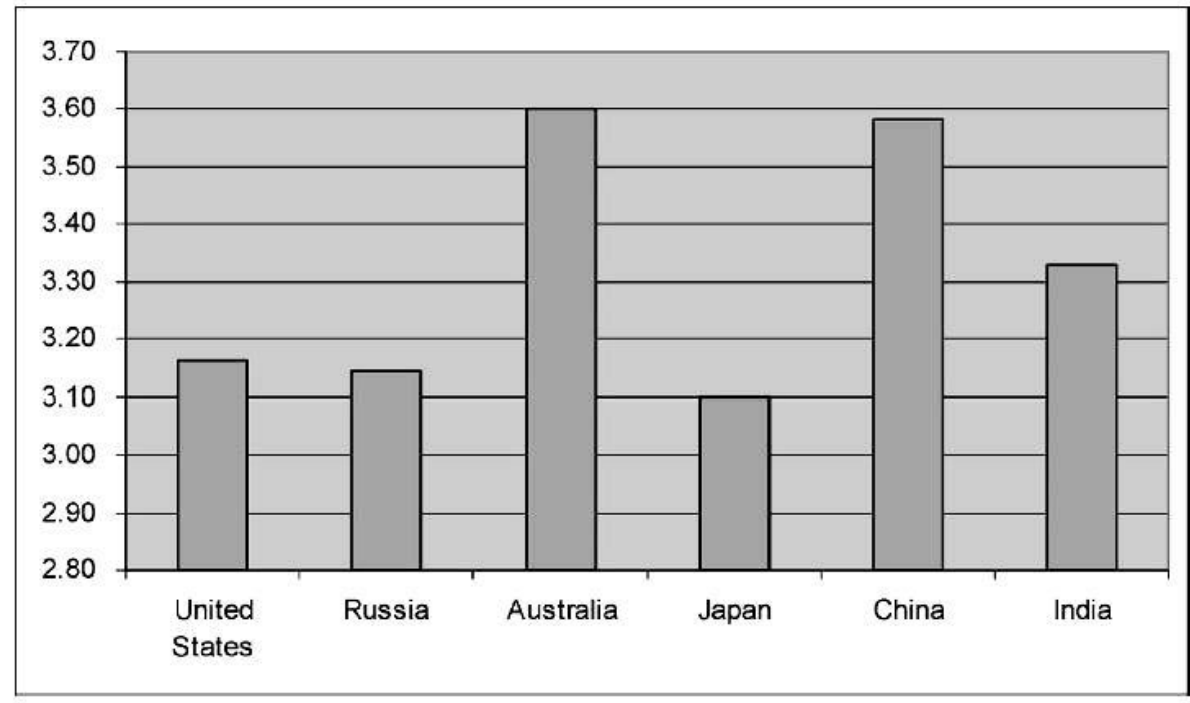

Wang, 2010: 62

Cependant, le modèle occidental de démocratie multipartite n'est peut-être pas aussi impartial qu'il est vendu à l'opinion publique internationale. Par exemple, on peut légitimement se demander si le mépris des droits humains fondamentaux dans le système de castes indien compte moins que la démocratie. Si l'on demandait à la population concernée de donner son avis, le tableau serait probablement différent (cf. graphique 11). On pourrait se demander, par exemple, si le système rigide des castes indiennes, les inégalités flagrantes, la pauvreté et le racisme en Inde sont des violations moins importantes des droits humains fondamentaux.

Certes, il existe des variations considérables dans l'évaluation de la performance du gouvernement au sein de grands pays comme l'Inde et la Chine, où les citoyens de différentes régions donneraient sans aucun doute des évaluations différentes de la bonne gouvernance. En outre, il existerait un écart de satisfaction entre les riches et les pauvres, ainsi qu'entre les 
populations côtières et de l'arrière-pays. Des découvertes récentes du «Ash Center for Democratic Governance and Innovation » de la « Harvard Kennedy School of Government », basées sur une enquête d'opinion publique à long terme en Chine, ont révélé que les citoyens chinois avaient une très grande satisfaction de 95,5\% des personnes interrogées auprès du gouvernement central en 2016. La satisfaction des citoyens américains vis-à-vis du gouvernement fédéral américain n'était au contraire que de $38 \%$ (Harsha, 2020). D'autres études universitaires ont suggéré par ailleurs de fortes divergences entre les propres évaluations des personnes, par rapport à l'évaluation de la qualité de la gouvernance telle que l'indice d'efficacité du gouvernement de la Banque mondiale (Wang, 2010). Ainsi, l'évaluation de la performance du gouvernement en Chine par les citoyens a été notée nettement plus élevée qu'en Inde.

Graphique 12 : Indicateurs de gouvernance dans le monde, 2020
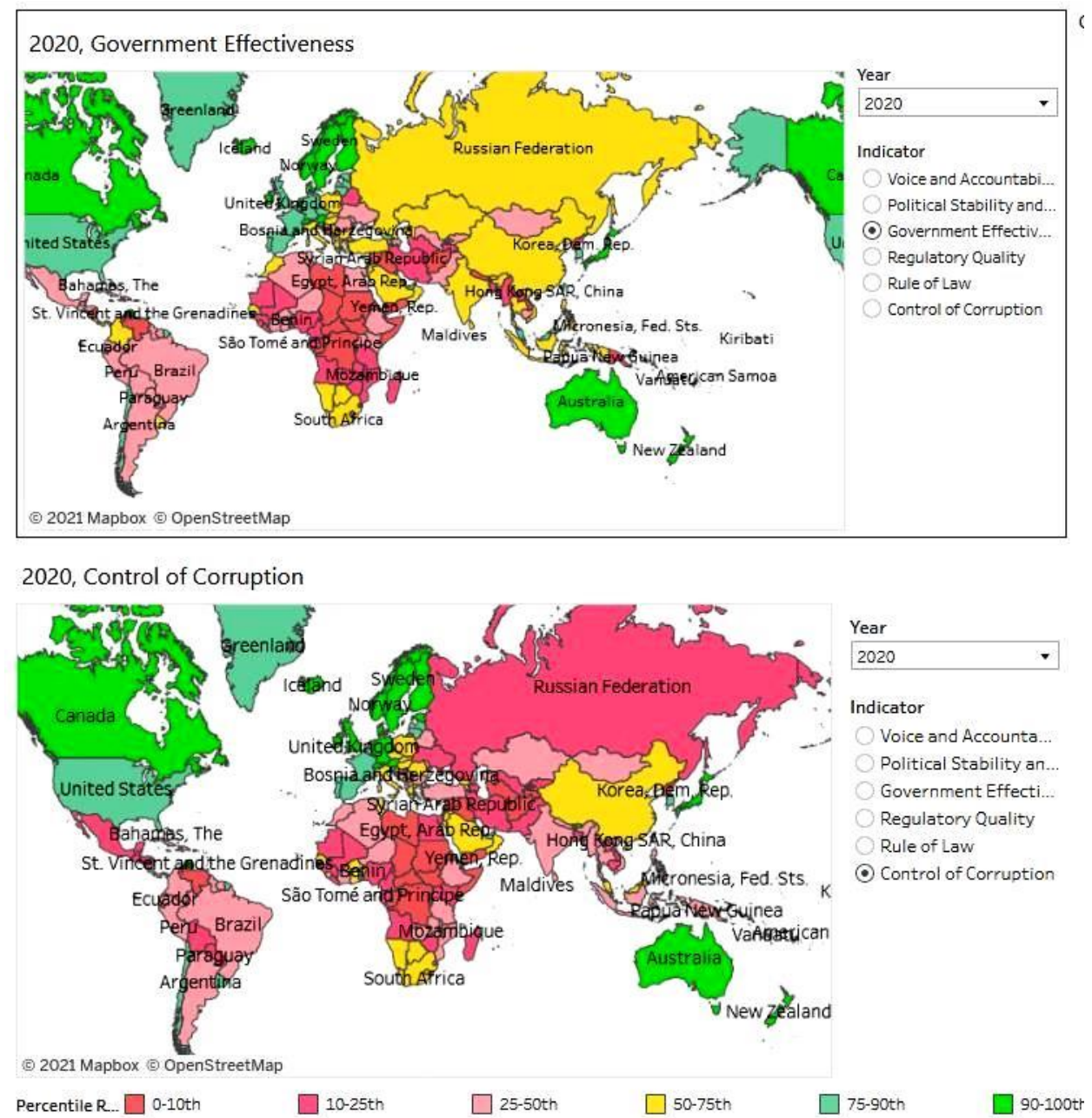

Source: Banque mondiale, 2021 
Graphique 13 : Indicateurs de gouvernance, Asie de l'Est, 2010 - 2020

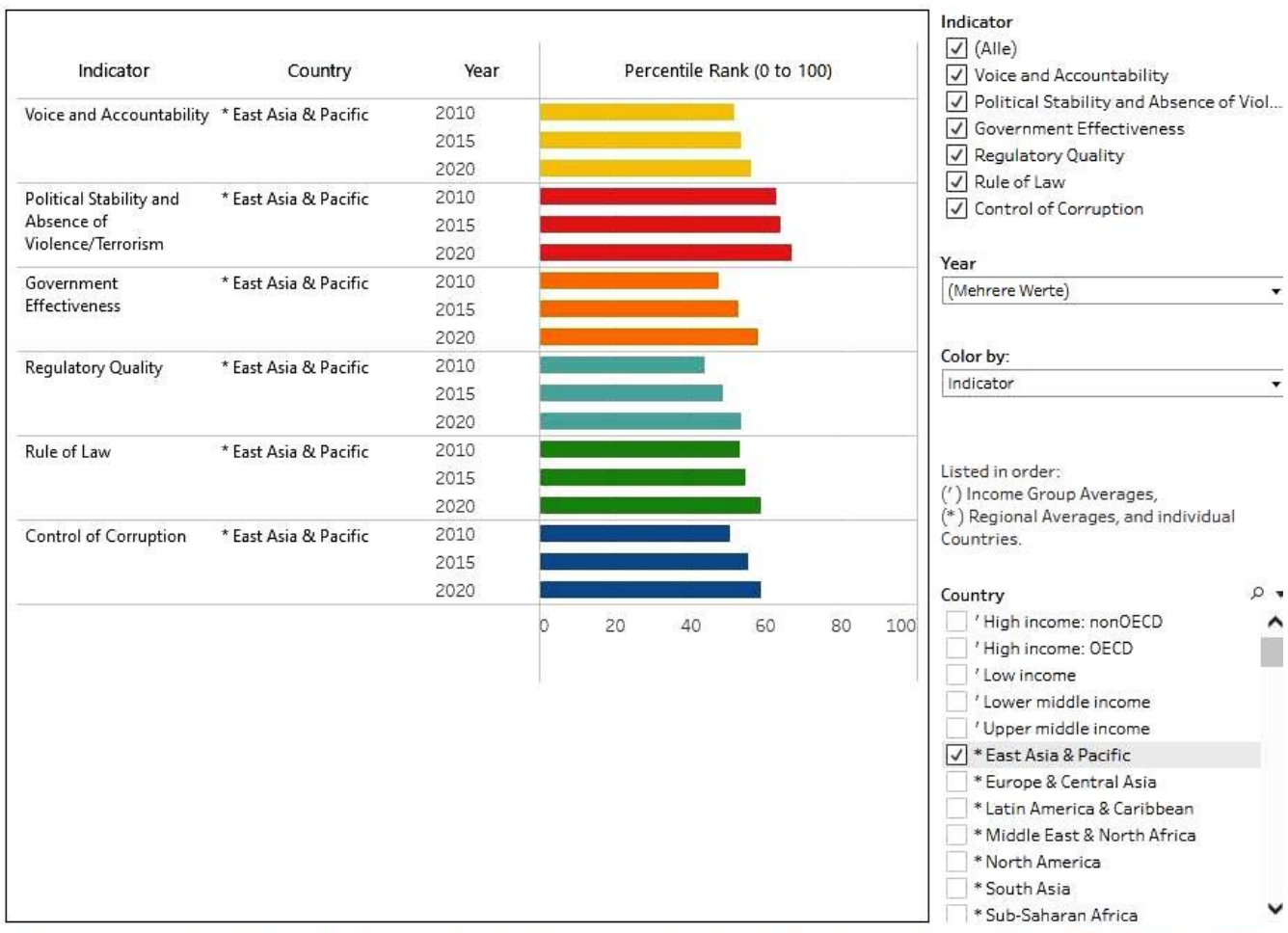

Source: Kaufmann D., A. Kraay, and M. Mastruzzi (2010), The Worldwide Governance Indicators: Methodology and Analytical Issues - https://papers.ssrn.com Isol3/papers.cfm?abstract id=1682130

Source: Banque mondiale, 2021

Graphique 14 : Indicateurs de gouvernance, Chine, 2010 - 2020

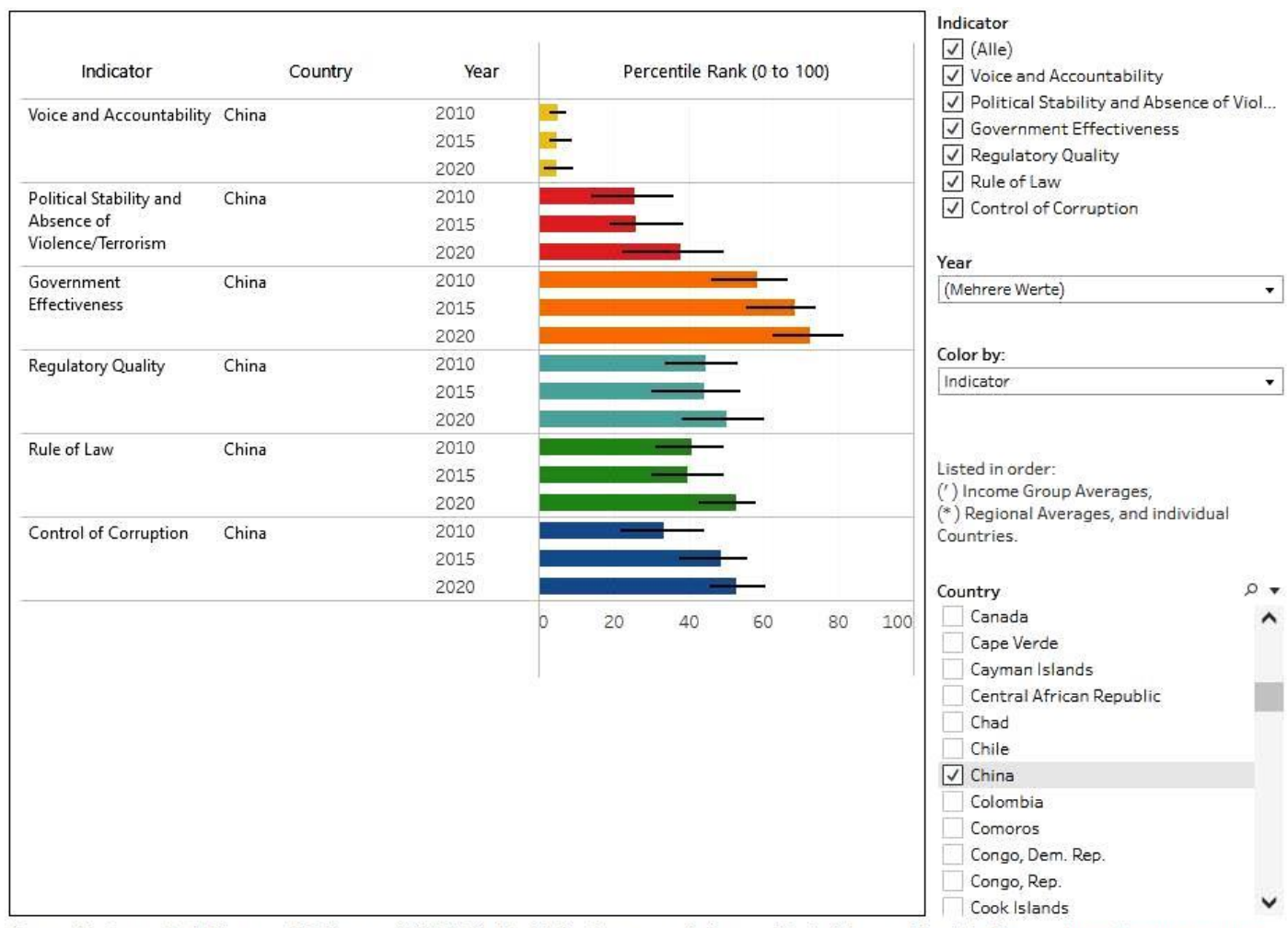

Source: Kaufmann D., A. Kraay, and M. Mastruzzi (2010), The Worldwide Governance Indicators: Methodology and Analytical Issues - httips://papers.ssm.com /so/3/papers.cfm?abstract_id $=1682130$

Source: Banque mondiale, 2021 
Aussi, en matière de réduction de la pauvreté, la Chine, qui compte entre-temps parmi les pays à revenu intermédiaire supérieur, fait mieux que l'Inde, cette dernière faisant toujours partie des pays à revenu intermédiaire inférieur selon le classement de la Banque mondiale. Cela peut être dû à la politique plus prononcée de Pékin pour lutter contre la pauvreté, mais aussi aux effets généraux de la stratégie de développement plus orientée vers les exportations de la Chine, entraînant une industrialisation et une urbanisation plus rapides que l'Inde. Pour réduire la pauvreté, une tâche primordiale des gouvernements des pays en développement, l'administration devrait donner la priorité à la création d'emplois dans les industries secondaires et tertiaires grâce à l'industrialisation et à la mondialisation afin d'absorber la main-d'œuvre agricole excédentaire, contribuant ainsi à réduire la pauvreté dans les zones rurales (Zhang, et al, 2020). Ainsi, au cours de la première année de la pandémie de Corona en Inde, environ 75 millions de personnes sont tombées dans la pauvreté, ce qui représente $60 \%$ de l'augmentation mondiale de la pauvreté, alors que la Chine n'a dû admettre qu'un million de pauvres supplémentaires. Le nombre total de pauvres Indiens s'élève désormais à 134 millions (Kapur, 2021).

Pourtant, des acteurs mondiaux asiatiques concurrents, comme l'Inde, ont non seulement de mauvais résultats dans la réduction de la pauvreté, mais ont également cultivé un racisme encore plus prononcé que la Chine, y compris la montée de l'afrophobie (Kohnert, 2021). Ainsi, toutes choses égales par ailleurs, la Chine pourrait avoir à l'avenir un avantage concurrentiel dans la quête mondiale des ressources et des marchés de l'Afrique, car les gouvernements africains réagissent de manière assez sensible au racisme dans les relations étrangères internationales et le commerce international. 


\section{References}

AITakarli N.S. (2020): China's response to the COVID-19 outbreak: A model for epidemic preparedness and management. Dubai Medical Journal, 2020;3:44-49

Ajl, Max (2010): Reclaiming Africa: scramble and resistance in the 21st century. Book Review, Review of African Political Economy, vol. 47:165, pp. 504-506

Asiedu, Kwasi Gyamfi (2020): After enduring months of lockdown, Africans in China are being targeted and evicted from apartments. Quartz Africa, 11 April 2020

Bodomo, Adams et al (2020): How African migrants in China cope with barriers to health care. The Lancet Public Health, vol. 5, issue 4, e192, April 01, 2020

Bräutigam, D., \& T. Xiaoyang (2011). African Shenzhen: China's special economic zones in Africa. Journal of Modern African Studies, 49(1), 27-54

Carling, Jørgen \& Heidi Østbø Haugen (2021): Circumstantial migration: how Gambian journeys to China enrich migration theory, Journal of Ethnic and Migration Studies, 47:12, 2778-2795,

Castillo, Roberto (2020): "Race" and "racism" in contemporary Africa-China relations research: approaches, controversies and reflections. Inter-Asia Cultural Studies, vol. 21 (3), pp. 310-336

Castillo, Roberto (2016): Of washing powder, Afrophobia and racism in China. The Conservation, 11 August 2016 (reprint in: Quartz Africa, 14 August 2016

Cheng, Y. (2019): Discovering China in Africa: Race and the Chinese perception of Africa and black peoples. In: Cheng, Yinghong (ed.)(2019): Discourses of Race and Rising China, pp. 161-237

Cheng, Y. (2011): From campus racism to cyber racism: discourse of race and Chinese nationalism. The China Quarterly, 207, pp. 561-579

Cissé, Daouda (2021): As migration and trade increase between China and Africa, traders at both ends often face precarity. Washington D.C.: Migration Policy Institute (MPI), July 21, 2021

Cissé, Daouda (2013): South-south migration and Sino-African small traders: A comparative study of Chinese in Senegal and Africans in China. African Review of Economics and Finance, Vol. 5 No. 1 (2013)

Cheuk, Ka-Kin (2019): Editorial - Transient migrants at the crossroads of China's global future. Transitions: Journal of Transient Migration, vol. 3, number 1, 1 March 2019, pp. 3-14(12)

Connor, Phillip (2018): At Least a Million Sub-Saharan Africans Moved to Europe Since 2010. March 22, 2018

Dalibi, Gidado, S. \& Bello S. Bello (2017): Accelerating the world's research. Socio-economic impacts of Chinese government-financed infrastructural development projects in Nigeria. Advances in Economics, Business and Management Research, Volume 33, pp. 579-588, Academia

Dever, James \& Jack Dever (2021): Information age imperialism: China, 'race,' and neo-colonialism in Africa and Latin America. Race, Racism and the Law, University of Dayton School of Law, website, 26 June 2021

Elochukwu, Anas (2016): Guangzhou's African Migrants: Implications for China's Social Stability and China-Africa Relations. Researchgate, December 2016 
Galchu, J. (2018): The Beijing consensus versus the Washington consensus: The dilemma of Chinese engagement in Africa. African Journal of Political Science and International Relations, Vol.12(1), pp. 1-9

Gazibo, Mamoudou \& Olga Alexeeva (2012): Socio-Demographic Profile of Africans in China. In: The African Community in China in the Age of Renewed China-Africa Cooperation. Ithaca New York: The Cornell Institute for African Development (IAD), Chapt: Socio-Demographic Profile of Africans in China

Giese, Karsten \& Laurence Marfaing (eds.) (2019): Chinese and African entrepreneurs: social impacts of interpersonal encounters. Leiden: Brill

Haifang, Liu (2021): Mapping the new migrants between China and Africa: theoretical and methodological challenges. In: Peilin, Li \& Laurence Roulleau-Berger (eds.): China's Internal and International Migration. London: Routledge, 16 August 2012, p. 11

Haugen, H. Ø. (2012): Nigerians in China: A second state of immobility. International Migration, 2012, vol. 50 (2), pp. $65-80$

Haugen, Heidi Østbø (2019): China-Africa exports: Governance through mobility and sojourning. Journal of Contemporary Asia, vol. 49:2, 294-312

Harsha, Dan (2020): Taking China's pulse. The Harvard Gazette, 9 July 2020

Ho, E. L. E. (2016). The geo-social and global geographies of power: Urban aspirations of 'worlding'African students in China. Geopolitics, vol. 22(1), pp. 15-33

Huang, Guangzhi (2019): Policing blacks in Guangzhou: How public security constructs Africans as Sanfei. Modern China, 2019, vol. 45, Number 2, p. 171 First Published July 19, 2018

IISD (2021): Chinese investment in Africa rises as project values and bilateral trade decline. Winnipeg, Manitoba, Canada, International Institute for Sustainable Development, 25 October 2021

Jayasuriya, S de Silva (2009): African identity in Asia: cultural effects of forced migration. African Diaspora Archaeology Newsletter, vol. 12, issue 3, September 2009

Jordan, Lucy \& Andrew Pau Hoang \& Cheryl H. K. Chui \& Wei Wang \& Valentina Mazzucato (2021): Multiple precarity and intimate family life among African-Chinese families in Guangzhou, Journal of Ethnic and Migration Studies, 47:12, 2796-2814

Kalantaryan, S \& McMahon, S (2020): Covid-19 and Remittances in Africa. Luxembourg: European Commission, Joint Research Centre (JRC) technical report, EUR 30262 EN

Kapur, Manavi (2021): India contributed to nearly $60 \%$ of the global rise in poverty in 2020. Quartz India, 18 March 2021

Kohnert, Dirk (2021): African migrant's plight in India: Afrophobia impedes India's race for Africa's resources and markets. MPRA-paper. No. 111077

Kohnert, Dirk (2016): Chinese and African migrant entrepreneur's articulation shaped by African agency. Strategic Review for Southern Africa, vol. 38 2016.2: 156-166

Kohnert, D. (2010): Are Chinese in Africa more innovative than Africans? Comparing Cultures of Innovation of Chinese and Nigerian entrepreneurial migrants. Hamburg: GIGA-WP, No. 140 
Kohnert, D. ( (2007): African migration to Europe: Obscured responsibilities and common misconceptions. GIGA-Working Paper, No. 49, Hamburg, May 2007

Kuo, Lily (2016): African migrants are returning from China and telling their compatriots not to go. Quartz Africa, July 1, 2016

Lan, Shanshan (2014): State regulation of undocumented African migrants in China: A multi-scalar analysis. Journal of Asian and African Studies, vol. 50, issue 3, 2015, first published 6 May 2014

Lan S. (2016): Between mobility and immobility: undocumented African migrants living in the shadow of the Chinese state. In: Wang D., He S. (eds), Mobility, sociability and well-being of urban living. Springer, Berlin, Heidelberg

Lan, Shanshan (2017): Mapping the new African diaspora in China. In; Race and the Cultural Politics of Belonging. New York: Routledge, Researchgate

Leslie, Agnes N. (2018): China-Africa Relations: Theoretical and Practical Perspectives on African "Migrants" in China (Introduction). African Studies Quarterly, vol. 17, Issue 4, February 2018

Liang, Kelly \& Philippe Le Billon (2020): African migrants in China: space, race and embodied encounters in Guangzhou, China. Social \& Cultural Geography, vol. 21:5, 602-628

Liu, Philip H. P. (2018): Finding the Baoding villages: Reviewing Chinese conceptualisation of SinoAfrican agricultural cooperation. Africa Spectrum, vol. 53 (29), pp. 91-118

Lin, Lavinia et al. (2015): Ebola outbreak: From the perspective of African migrants in China. American Journal of Public Health; Washington Bd. 105, Ausg. 5, (May 2015): E5-E6

Lisimba, Alpha Furbell (2020): China's trade and investment in Africa - Impact on development, employment generation \& transfer of technology. Springer Singapore

Lufrano, Richard (1992): Nanjing spring: The 1989 student movement in a provincial capital. Bulletin of Concerned Asian Scholars, vol. 24 (1), pp. 19-42

Lyons, M. \& A. Brown \& Z. Li (2008): The 'third tier' of globalization: African traders in Guangzhou. City, Analysis of Urban Change, Theory, Action, vol. 12, 2008 - Issue 2

Mbamalu, Socrates (2018): Plight of African workers under Chinese employers. African Liberty, 27 September 2018

McGreal, Chris (2007): Mbeki criticised for praising 'racist' Sarkozy. The Guardian, 27 August 2007

Marfaing, Laurence \& /Alena Thiel (2014): "Agents of translation": West African entrepreneurs in China as vectors of social change. DFG WP series, No. 4, academia.edu

Marfaing, Laurence \&/ Alena Thiel (2015): Networks, spheres of influence and the mediation of opportunity: the case of West African trade agents in China. Journal of Panafrican Studies, vol. 7, Number 10, pp. 1-20

McDonell, Stephen (2021): Why China is still trying to achieve zero Covid. BBC News, Beijing, 15 November 2021

Mohan, Giles \& Ben Lampert (2013): Negotiating China: Reinserting African agency into ChinaAfrica relations. African Affairs, vol. 112, Issue 446, January 2013, pp. 92-110 
Müller, A. \& R. Wehrhahn (2011): New migration processes in contemporary China-The constitution of African trader networks in Guangzhou. Geographische Zeitschrift, 2011, vol. 99, issue $2 / 3$, pp. 104-122

Mulvey, Benjamin (2021): "Decentring" international student mobility: The case of African student migrants in China. Population, Space and Place, vol. 27, Issue 3, April 2021 --First online: October 2020

Mupambwa, G. \& M. B. Xaba (2021): 'Investors' or looters? A critical examination of mining and development in Africa. Chapt. 10, in: Warikandwa, T.V. et al. (eds.): Grid-locked African economic sovereignty. Bamenda: Langaa Research \& Publishing Common Initiative Group (Cameroon)

Nawyn, Stephanie J. (2016): Migration in the global South: Exploring new theoretical territory. International Journal of Sociology, vol. 46 (2), pp. 81-84

Okereby, Austine (2019): Specialized Economic Zones (SEZs) in Africa: Exporting Production, Performance, and Perils. Business, Politics \& Foreign Affairs, impakter.com, 16 August 2019

Okon, Enoch Ndem \&Victor Ojakorotu (2021): Chinese Resource Policy and Human Security in Africa: A Discourse. In: Ani, K. J. \& V. Ojakorotu \& K. Bribena (eds.): Political Economy of Resource, Human Security and Environmental Conflicts in Africa. London: Palgrave MacMillan, pp. 13-38, 1 September 2021

Parashar, Swati \& Michael Schulz (2021): Colonial legacies, postcolonial 'selfhood' and the (un)doing of Africa, Third World Quarterly, 42:5, 867-881

Perrot, Sandrine \& Dominique Malaquais (2009): Penser l'Afrique à l'aune des globalisations émergentes. Politique africaine, vol. 113, no. 1, pp. 5-27.

Pomfret, John (1989): China claims Africans confess, boycott continues in Beijing. AP-news, 6 January 1989

PTI (2016): Western media magnifying racism in China over racial Ad: Experts. The Economic Times News, indiatimes.com, 30 May 2016

Ramachandaran, Shastri (2018): Afrophobia and racism keep India trailing behind China. IDN - indepth news.net, 18 September 2018

Rotberg, Robert I. (2017): How Chinese traders out-compete in Africa. China \& US Focus, 23 May 2017, Harvard Kennedy School

Sasu, Doris Dokua (2021): Number of Chinese migrants in African countries 2000-2019. Statistica, 25 August 2021

Sautman, B., \& Y. Hairong (2009): African perspectives on China-Africa links. The China Quarterly, 199, 728-759

Servant, Jean-Christophe (2005): Moscow and Beijing, Asia's roaring economies - China's trade safari in Africa. Le Monde Diplomatique, May 2005

Sibiri, Hagan (2021): Present and emerging issues detrimental to Ghana-China relations. In: Amoah, Lloyd (ed.): Sixty Years of Ghana-China Relations: Friendship, Friction, and the Future. Centre for Asian Studies, University of Ghana Press, June 2021

Song, G. \& L. Liu \& S. He \& L. Cai \& C. Xu (2020): Safety perceptions among African migrants in Guangzhou and Foshan, China. Cities, Volume 99, April 2020 
Stein, Peter \& Emil Uddhammar (2021): China in Africa: The Role of Trade, Investments, and Loans Amidst Shifting Geopolitical Ambitions. Observer Research Foundation, ORF Occasional Paper No. 327, August 2021

Sylvanus, Nina (2013): Chinese devils, the global market, and the declining power of Togo's NanaBenzes. African Studies Review, vol. 56, Issue 1, pp. 65-80

UNCTAD (2020): World Investment Report for 2020 - International production beyond the pandemic. Geneva: 2020

Wang, Zhengxu (2010): Citizens' satisfaction with government performance in six Asian-Pacific giants. Japanese Journal of Political Science, vol. 11 (1), 26 February 2010

Wilczak, Jessica (2018): "Clean, safe and orderly": Migrants, race and city image in global Guangzhou. Asian and Pacific Migration Journal, Vol 27, Issue 1, 2018, March 19, 2018

Xiaoyang, Tang (2016): Does Chinese employment benefit Africans? Investigating Chinese enterprises and their operations in Africa. African Studies Quarterly, vol. 16, Issue 3-4, December 2016

Xu, T (2013): The social relations and interactions of black African migrants in China's Guangzhou province. In: Li, Peilin \& Laurence Roulleau-Berger (2013): China's internal and international migration. New York: Routledge, 2013, China policy series, 27

Yin, S. (2017): Network, Mobility, and Integration Undocumented African Migrants in Guangzhou, China. WIDER Development Conference: Migration and Mobility Accra, Ghana, 5 October 2017

Zeleza, Paul Tiyambe (2008): Dancing with the Dragon Africa's Courtship with China. The Global South, Vol. 2, No. 2, Fall, 2008, pp. 171-187

Zhang, T. \& Y. Zhang \& G. Wan \& H. Wu (2020): Poverty reduction in China and India: a comparative study. The Singapore Economic Review, vol. 65, (1), pp. 95-115

Zhou, Min \& Shabnam Shenasi \& Tao Xu (2016): Chinese Attitudes toward African Migrants in Guangzhou, China. International Journal of Sociology, vol. 46:2, 141-161

Zhou, Min \& Tao Xu \& Shabnam Shenasi (2016a): Entrepreneurship and interracial dynamics: a case study of self-employed Africans and Chinese in Guangzhou, China, Ethnic and Racial Studies, 39:9, 1566-1586 


\section{Abstract : African migrants plight in China: Afrophobia impedes China's race for Africa's resources and markets}

Discrimination against the approximately 500,000 African (mostly irregular) immigrants has recently spread in China. During the corona pandemic, it degenerates into a true Afrophobia. Shortly before, five Nigerians in Guangzhou had reportedly tested positive for Covid-19. Africans are widely accused as drug traffickers and criminals. Also, they would endanger China's global competitiveness for Africa's resources through media baiting abroad. Current reports testify the displacement of African migrants from homes and hotels in Guangzhou (Canton), where most of the Africans live. They are dependent on informal, mostly illegal networks in order to be able to stay in the country. In online social networks Afrophobia as cyber racism is particularly pronounced. Thereby, racism is more deeply rooted in the mentality of many Chinese than is commonly assumed. According to a traditional Chinese proverb, the greatest evil to be avoided is 'the destroyed nation and the annihilated race'. In addition, since 2005 land-grabbing by Chinese entrepreneurs in sub-Saharan Africa arose international attention. Its main purpose is to ensure food security in China and to profit from international grain speculation. It was racially legitimized from the start, with slogans such as, only Chinese investments could save Africans from their traditional 'laziness'. This repeats deeply rooted neo-colonial European prejudices of a 'wild, ahistoric and uncivilized Africa'. The prejudices are still associated with a feeling of racial superiority. The social fabric of China has always embodied essential characteristics of the exclusion of 'foreigners' focused on ethnicity, race, religion, sexual orientation and gender. The African Union, various African governments and even the United States have sharply criticized Beijing for mistreating migrants, particularly those from Nigeria, Ghana, Kenya and Uganda. Racist attacks on Africans in China have an oppressively long tradition, associated with the expansion of bilateral Chinese petty trade in sub-Saharan Africa in the early 2000s and the subsequent influx of African petty traders into China.

\section{Zusammenfassung: Die Misere afrikanischer Migranten in China: Afrophobie behindert Chinas Rennen um Afrikas Ressourcen und Märkte}

In China breitet sich in letzter Zeit eine Diskriminierung der ca. 500.000 afrikanischen (meist irregulären) Einwanderer aus. Sie artet in der Corona-Pandemie zu einer wahren Afrophobie aus. Afrikaner werden pauschal als Drogenhändler und Kriminelle beschuldigt. Sie würden zudem über Medienhetze im Ausland Chinas globale Wettbewerbsfähigkeit um Afrikas Ressourcen gefährden. Aktuelle Meldungen berichten von der Vertreibung afrikanischer Migranten aus Wohnungen und Hotels in Guangzhou (Canton), wo die meisten Afrikaner leben. Sie sind auf informelle, meist illegale Netzwerke angewiesen, um im Land bleiben zu können. In sozialen online Netzwerken ist Afrophobie als Cyber-Rassismus besonders ausgeprägt. Rassismus ist in der Mentalität vieler Chinesen tiefer verwurzelt als gemeinhin angenommen. Nach einem traditionellen chinesischen Sprichwort gilt als größtes zu vermeidendes Übel die ,zerstörte Nation und die vernichtete Rasse“. Hinzu kommt seit 2005 die ,Landnahme“ chinesischer Unternehmer in Schwarzafrika. Sie dient hauptsächlich der Absicherung der Nahrungsmittelsicherheit Chinas und der internationalen Getreidespekulationen. Sie wurde von Anbeginn rassistisch legitimiert, mit Sprüchen, wie beispielsweise, nur chinesische Investitionen könnten die Afrikaner vor ihrer tradierten „Faulheit" retten. Damit wiederholen sich tief verwurzelte neo-koloniale europäische Vorurteile von einem ,wilden und unzivilisierten Afrika“. Dies ist nach wie vor verbunden mit einem Gefühl rassischer Überlegenheit. Das soziale Gefüge Chinas verkörperte schin immer wesentliche Merkmale der Ausgrenzung von „Fremden“. Fokkussiert auf ethnische Zugehörigkeit, Rasse, Religion, sexuelle Orientierung und Geschlecht. Die Afrikanische Union, verschiedene afrikanische Regierungen und sogar die Vereinigten Staaten haben Peking wegen der Misshandlung von Migranten, vor allem aus Nigeria, Ghana, Kenia und Uganda, scharf kritisiert. Kurz zuvor waren Berichten zufolge fünf Nigerianer in Guangzhou positiv auf Covid-19 getestet worden. Rassistische Angriffe auf Afrikaner in China haben eine bedrückend lange Tradition, die mit der Ausweitung des bilateralen Kleinhandels von Chinesen in Subsahara-Afrika Anfang der 2000er und dem anschließenden Zustrom afrikanischer Kleinhändler nach China zusammenhängt. 


\section{非洲移民在中国的困境： \\ 针对非洲人的种族主义阻碍中国争夺非洲资源和市场}

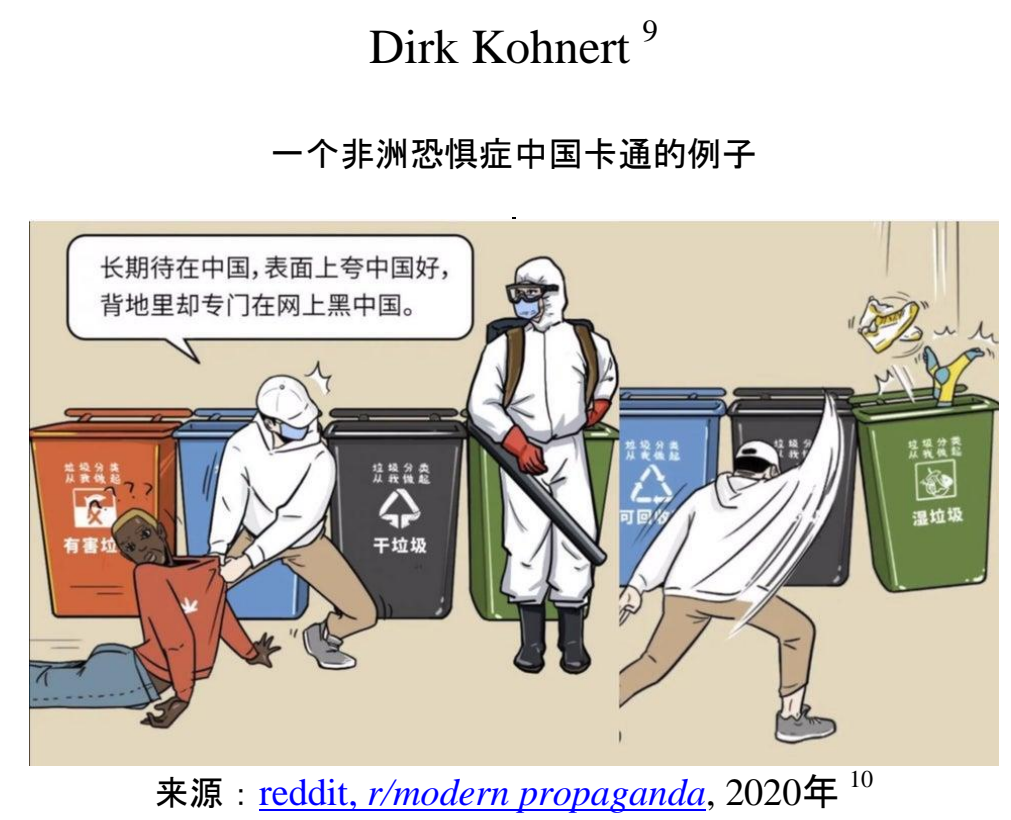

对大约 500,000 名非洲移民（主要是非正规移民）的歧视最近在中国蔓延。在电晕大流 行期间, 它退化为真正的非洲恐惧症。不久之前, 据报道, 广州的五名尼白利亚人的 Covid-19 检测呈阳性。非洲人被广泛指责为毒贩和罪犯。此外, 它们还会通过国外媒体 的引诱，危及中国对非洲资源的全球竞争力。目前的报告证实了非洲移民众大多数非洲 人居住的庯 (广州) 的家庭和酒店流离失所。他们依靠非正式的、主要是非法的网络 才能留在国内。在在线社交网络中, 非洲恐惧症作为网络种族主义尤为奛显。因此, 种 族主义在许多中国人的心态中根深蒂固, 而不是通常的假设。中国有句浐语, 最大的恶 是“亡国灭族”。此外, 自2005年以来, 中国企业家在撒哈拉以南非洲的主地掠夺引起了 国际养注。其主要目的是确保中国的粮食安全, 并从国际粮食投机中获利。它从一开始 就在种族上合法化，口号是，只有中国的投资才能将非洲人众传统的“濑惰”中㧺救出莱 。这重复了根深蒂固的新殖民主义欧洲对“狂野、非历史和未开化的非洲”的偏见。偏见 仍然与种族优越感有养。中国的社会结构始终体现着排斥“外国人”的基本特征, 侧重于 民族、种族、宗教、性取向和性别。非洲联盟、非洲各国政府甚至美国都严厉批评北京 虐待移民，尤基是来自尼白利亚、加纳、肯㞾亚和乌早达的移民。在中国，针对非洲人 的种族主义攻击有着悠久的传统, 这与 2000 年代初期中国在撒哈拉以南非洲的双边小额 贸易扩张以及随后菲洲小商贩浦入中国有。

关键词: 中国、非洲、国际移民、仇外心理、仇外心理、种族主义、政治暴力、中非关系、非 正规部门、非法移民、强迫移民、奴隶贸易、少数民族、汇款

JEL 代码: F16、F22、F24、F51、F54、I24、I31、J46、J61、N15、N35、O15、O17 、O53、Z13

\footnotetext{
${ }^{9}$ Dirk Kohnert, 汉堡 GIGA 非洲事务研究所副所长（已退休）。草案 : 2022 年 1 月 13 日

10 发表者 u/friend $1 \mathrm{y}$, 发布, 2020 年, 谴责一部具有非洲恐惧症和种族主义的排外中国漫画, 标题为: “长期留在中 国; 在人们面前赞美中国，而在每个人背后在互联网上谈论中国的狗屎”。 OEPEQY 指的是“附加上下文：“涂 抹 (某人) “一词, 在此翻译为“谈论狗屎”, 是 “黑 “, 这也意味着“黑色”。这可能是种族文字游戏的一种形式” (来 源：OEPEQY, reddit, r/modern propaganda, 2020年). 这幅漫画是指中国业主和市政当局在经历了数月的封锁后将非 洲租户赶出公寓的野蛮政策 (Asiedu, 2020).
} 